\title{
Modeling and optimization of multimodal urban networks with limited parking and dynamic pricing
}

\author{
Nan Zheng, Nikolas Geroliminis* \\ Urban Transport Systems Laboratory, École Polytechnique Fédérale de Lausanne (EPFL), Route Cantonale, 1015 Lausanne, Switzerland
}

\section{A R T I C L E I N F O}

\section{Article history:}

Received 29 March 2015

Revised 20 October 2015

Accepted 22 October 2015

Available online 11 December 2015

\section{Keywords:}

Public transport

MFD

Pricing

Cruising for parking

Optimization

\begin{abstract}
A B S T R A C T
Cruising-for-parking constraints mobility in urban networks. Car-users may have to cruise for on-street parking before reaching their destinations. The accessibility and the cost of parking significantly influence people's travel behavior (such as mode choice, or parking facility choice between on-street and garage). The cruising flow causes delays eventually to everyone, even users with destinations outside limited parking areas. It is therefore important to understand the impact of parking limitation on mobility, and to identify efficient parking policies for travel cost reduction. Most existing studies on parking fall short in reproducing the dynamic spatiotemporal features of traffic congestion in general, lack the treatment of dynamics of the cruising-for-parking phenomenon, or require detailed input data that are typically costly and difficult to collect. In this paper, we propose an aggregated and dynamic approach for modeling multimodal traffic with the treatment on parking, and utilize the approach to design dynamic parking pricing strategies. The proposed approach is based on the Macroscopic Fundamental Diagram (MFD), which can capture congestion dynamics at network-level for single-mode and bi-modal (car and bus) systems. A parsimonious parking model is integrated into the MFD-based multimodal modeling framework, where the dynamics of vehicular and passenger flows are considered with a change in the aggregated behavior (e.g. mode choice and parking facility choice) caused by cruising and congestion. Pricing strategies are developed with the objective of reducing congestion, as well as lowering the total travel cost of all users. A case study is carried out for a bi-modal city network with a congested downtown region. An elegant feedback dynamic parking pricing strategy can effectively reduce travel delay of cruising and the generic congestion. Remarkably, such strategy, which is applicable in real-time management with limited available data, is fairly as efficient as a dynamic pricing scheme obtained from system optimum conditions and a global optimization with full information about the future states of the system. Stackelberg equilibrium is also investigated in a competitive behavior between different parking facility operators. Policy indications on on-street storage capacity management and pricing are provided.
\end{abstract}

(c) 2015 Elsevier Ltd. All rights reserved.

\section{Introduction}

Cruising-for-parking can significantly influence mobility in congested urban networks. In the city center where road space is limited, car-users may have to cruise for available parking spots before reaching their destinations. Cruising cause delays eventually to everyone, even users with destinations outside limited parking areas. For example, according to Shoup (2006), the

\footnotetext{
* Corresponding author. Tel.: +41 798103931.

E-mail addresses: nan.zheng@epfl.ch (N. Zheng), nikolas.geroliminis@epfl.ch (N. Geroliminis).
} 
average cruising delay can take up 30\% of the total trip time in American metropolitan regions. Furthermore, the accessibility and the cost of parking (parking fee) closely impact people's travel behavior, such as mode choice (cars or buses) or parking facility choice (on-street or garage parking). Therefore, the impact of parking on mobility should not be overlooked and it is crucial to understand traffic dynamics under parking limitations. This paper aims to make effort into this direction by developing a novel modeling approach to capture the performance of congested multimodal networks with parking constraint. The ultimate goal is to integrate this approach in an optimization framework to identify efficient parking management policies, for example a dynamic parking pricing that influences car user's mode choice reducing the total delays of all users.

Research in transport economics have proposed road pricing as an effective policy to relieve traffic congestion in cities for many years. Despite the vast scientific literature, a small number of cities have actually implemented congestion tolls due to social disagreement and political issues. As an alternative, on-street parking pricing can be an efficient policy, since parking is priced almost everywhere and its implementation is more straightforward compared with cordon or area-based pricing. The approach for determining prices should be consistent with appropriate modeling of traffic congestion and its dynamics. Pricing parking can be "imperfect" substitutes of first-best pricing schemes, as it is charged at the end of a trip and cannot easily differentiate trip length and routes travelled (Verhoef et al., 1995). Nevertheless, recent advancements in monitoring traffic with smart sensors combined with proper models of traffic congestion dynamics for multimodal systems, could improve the estimation of the real cost of cruising-for-parking and create efficient and socially-acceptable pricing policies. At the same time, traffic mobility in multimodal systems is inherently a distributed and interconnected process, which should be modeled and managed as a whole to improve the global operational efficiency while avoiding conflicting decisions. A commonly-used fully decentralized optimization approach for traffic management is not appropriate for heterogeneously loaded networks with multiple modes of transport, short links and spillbacks, resulting in the saturation of neighborhood networks (Haddad et al., 2013). In this work we propose an aggregated and dynamic approach for modeling multimodal traffic with the treatment on parking, and we utilize this approach to design dynamic parking pricing strategies.

Consider a system where travelling by car and searching for an on-street parking is the only available mode of transport (see for example Arnott and Rowse (2009), Geroliminis (2009), Arnott and Inci (2010), Geroliminis (2015) and Cao and Menendez (2015b)). Under high traveling and thus parking demand, the system reaches to a stable traffic state which is close to gridlock. Parking will always be full and whenever there is a free spot, it will be occupied immediately by the cruising vehicles. If users (passengers) have efficient alternative choices (e.g. changing departure time, utilizing a parking garage, switching to public transport) and some of these choices are properly priced, then the system might end up in non-gridlock equilibrium states. This paper investigates the traffic dynamics of a system with limited on-street parking, unlimited but priced garage parking and a public transport alternative. It develops a feedback-based pricing scheme that does not require prediction of the state of the system and shows that hyper-congestion and cruising can be avoided, almost equally well with a global optimization that requires information for the future states of the system. It also investigates how close such a traffic management strategy can get to the system optimum pricing with perfect information of the future conditions of the system. While detailed micro- or agent-based simulations could provide a scenario-based analysis of such a system, we follow a dynamic aggregated approach consistent with the physics of traffic congestion that can contribute to develop physical intuition for such a challenging problem. The approach focuses eventually on a monitoring-based control, where treatments of behavioral side, such as departure time, are not necessarily required. Furthermore, incorporating departure time choices will make the solution approach too hard for analytical derivations and is beyond the scope of the paper.

Extensive studies have been dedicated to address how parking and parking policies influence people's mode choice and travel delay. Representative works can be found in Arnott and Rowse (1999), Anderson and de Palma (2004, 2007), Arnott (2006), Forsgerau and dePalma (2013), Qian et al. (2013) and elsewhere. The foundation of these works was the classical bottleneck-model developed by Vickrey (1969), while researchers made important extensions of Vickrey's model by considering parking constraints to reveal behavioral changes under parking limitation and parking-related policies. However, few studies discussed the dynamic influence of parking on traffic flow congestion. In case of insufficient parking capacity, for instance, cruise-for-parking flows should be treated differently than the normal running flows. Existing analysis on this subject include for instance, Arnott and Rowse (2009) and Arnott et al. (2015), which took the impact of cruising into account when analyzing parking pricing policies; Martens et al. (2010) examined the effect of spatial distribution of parking spots on searching-for-parking behavior; Horni et al. (2013) incorporated parking choice and searching-for-parking behavior model in an agent-based framework with simplistic traffic model; He et al. (2015) derived equilibrium conditions where car users compete for limited parking spaces, and investigated optimal pricing strategy to drive a system optimum. The aforementioned papers fall short in describing traffic dynamics as they assume static traffic states without dealing with the dynamics of traffic (e.g. how traffic density changes over time) that can be significantly different in the presence of parking limitations. Recent works (Liu et al., 2014; Qian and Rajagopal, 2014) have incorporated modeling and pricing parking in the classical morning commute with constant capacity of a bottleneck, which is not a realistic representation of a downtown city with traffic congestion and overcrowding of on-street parking. The treatment on the physics of cruising-for-parking and the connection between cruising and congestion remain challenging research subjects, though several recent studies indeed moved toward this direction: Gallo et al. (2011) considered parking costs into account in their traffic assignment model; van Ommeren et al. (2012) carried out an empirical study and reported the diverse features of the cruising behavior of the Dutch drivers; while Guo and Gao (2012) developed a model to estimate travel time including the delay from searching for on-street parking place. These approaches either require data that is difficult to collect (e.g. detailed information of origin-destination and parking availability at destination) or become computationally expensive in large-scale applications (e.g. microscopic traffic or agent-based models) that do not allow for physical interpretation of the analysis. Several 


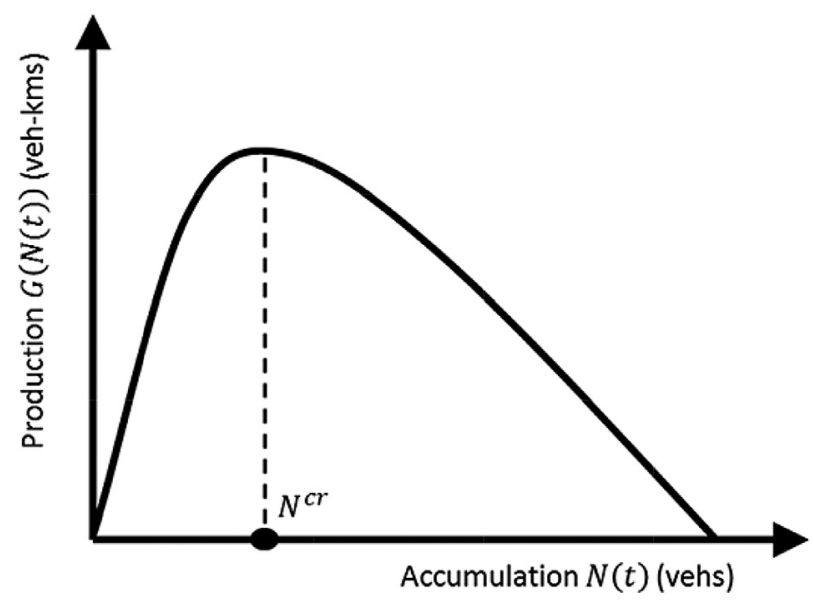

Fig. 1. A well-defined MFD for a single-mode network.

works analyzed the delay caused by on-street curb parking near intersections, see for example in Yousif and Purnawan (2004) and Cao and Menendez (2015a). Nevertheless, these models cannot be readily applied on large-scale networks either.

For large-scale network application, latest findings on the low-scattered Macroscopic Fundamental Diagram (MFD) show promising results in modeling the complex dynamics of urban congestion. An example MFD of a single-mode network (e.g. a caronly network) is displayed in Fig. 1, relating the production of the network (total vehicle kilometers travelled in during certain time $t$ and the vehicle accumulation in the network) number of vehicles inside the network during $t$. The critical accumulation $N^{c r}$ marks the maximum flow capacity that a network can provide, which is an important property of the network. We will show later that $\mathrm{N}^{\mathrm{cr}}$ significantly helps the development of traffic control strategies to manage congestion.

Geroliminis $(2009,2015)$ propose a macroscopic parking model, which is built into an MFD framework to capture the influence of parking on congestion. This work shows that the MFD-based model reflects the dynamics of parking flows in an urban network, only requiring data that can be practically obtained. Moreover, this work reveals that if cruising-for-parking is intense, demand which is lower than the network capacity can create significant congestion due to higher trip lengths. It proposes simple types of traffic management that alleviate the phenomenon of cruising, and shows that perimeter control (restricting the inflow to an inner zone by altering traffic signal settings) can significantly reduce delay if cruising-for-parking is not so intense and many trips are not originated from the inner zone; for networks where trips are generated everywhere, pricing is more efficient than signal control. What is still missing though is an integrated approach, which can be applied for multi-modal multi-region systems. Furthermore, the impact of spatial limitation and management strategies on multimodal mobility deserves further research effort. Although mode choice was indeed studied by taking into account the influence of parking availability and cost, see for example in Li et al. (2007), Zhou et al. (2008) and Liu et al. (2009), the utilized approaches again fall short in the treatment of cruising and congestion dynamics.

To summarize, the objective of this research is to develop an aggregated macroscopic approach for modeling and controlling multimodal systems with parking limitation. This approach shall enable the development and optimization of parking pricing strategies for improving multimodal mobility. A recent study by the authors presents a macroscopic approach to model the dynamics of a bi-modal (cars and buses) transport system (Zheng and Geroliminis, 2013) without any parking treatment. The work shows that effective management strategies such as allocation of dedicated-bus-lanes and area-based pricing can be developed by utilizing a low-scattered bi-modal MFD. A parking module will be integrated into this bi-modal modeling framework to model cruising-for-parking and estimate cruising delay, where the bi-modal traffic movement of traffic will be reproduced by specific treatment on the car flow families (moving, cruising-for-parking and parked). The pricing strategies range from simple constant pricing to complex full horizon optimization with perfect information for the system demand and mode choice of the users, which is expected to lead close to system optimum conditions. While this strategy is considered infeasible for real implementation, it provides a fair comparison for the efficiency of more realistic control strategies.

An elegant feedback time-dependent parking pricing strategy is developed with an objective to maximize the production of the network and to keep the cruising for on-street parking at low values. Remarkably, such strategy, which is applicable in real-time management with limited available data, is fairly as efficient as the full horizon optimization. Stackelberg equilibrium is also investigated in a competitive behavior between different parking facility operators. Our analysis also demonstrates that feedback pricing functions powerfully even when parking storage capacity decreases and is able to avoid high congestion and cruising.

The remaining part of the paper is organized as follows. Section 2 presents the methodology for modeling multimodal traffic system dynamics with parking, extending the work of Geroliminis (2009, 2015). Four subsections are dedicated respectively on the underlying traffic model, parking model, system model and choice model. Different parking pricing strategies are then developed in Section 3, with the objectives of reducing congestion and the total travel cost of all the users. Cooperative and 
Table 1

List of main variables and parameters.

\begin{tabular}{|c|c|c|}
\hline Variables & Units & Description \\
\hline$t$ & & Time interval index \\
\hline$m$ & & $\begin{array}{l}\text { Mode of transport } m \in\{o s, g, b\} \text { (indices represent car with on-street parking, car } \\
\text { with garage parking and bus respectively) }\end{array}$ \\
\hline$P_{i}^{m}(t)$ & veh-kms & Travel production by mode $m$ in region $i$ at $t$ \\
\hline$N_{i}^{m}(t)$ & vehs/region & Accumulation of vehicles of $m$ currently in $i$ \\
\hline$G_{i}^{m}\left(N_{j}^{m}(t)\right)$ & & Production-MFD of $m$ in $i, P_{i}^{m}$ as a function of $N_{i}^{m}$ \\
\hline$Q_{i}^{k m}(t)$ & vehs/t & $\begin{array}{l}\text { Travel demand generated in } i \text { and choosing } m \text {, with internal or external } \\
\text { destination } k\end{array}$ \\
\hline$O_{i \rightarrow j}^{k m}(t)$ & vehs/t & $\begin{array}{l}\text { Vehicular outflow (trip completion rate) of } m \text { from } i \text { to } j \text { with final destination } k, k \\
\neq i\end{array}$ \\
\hline$O_{i}^{m}(t)$ & vehs/min & Total outflow of $i, O_{i}^{m}(t)=\sum_{j} O_{i \rightarrow j}^{k m}(t)$ \\
\hline$I_{i}^{m}(t)$ & vehs/min & Total incoming flow of $m$ from the adjacent regions of $i$ to $i$ \\
\hline$N P_{i}^{m}(t)$ & pers & Accumulation of persons on $m$ currently in $i$ \\
\hline$O P_{i}^{m}(t)$ & pers/min & Total passenger outflow (trip completion of passenger flow) \\
\hline$N_{x, i}(t)$ & vehs & $\begin{array}{l}\text { Accumulation of car family } x \text { in } i(x=r, s, o, g, o s) \text { (indices represent running, } \\
\text { searching, outgoing, garage and on-street) }\end{array}$ \\
\hline$O_{x, i}(t)$ & vehs/min & Outflow of car family $x$ to its subsequent family \\
\hline$\omega_{o s, i}^{c}(t) \mid \omega_{g, i}^{c}(t)$ & $\%$ & Fraction of outflow of cars using on-street (or garage) parking \\
\hline$\omega_{i}^{m}(t)$ & $\%$ & Fraction of the generated demand in $i$ choosing travelling with $m$ \\
\hline$O_{r \rightarrow s, i}(t) \mid O_{r \rightarrow g, i}(t)$ & vehs/t & $\begin{array}{l}\text { Vehicular transfer flow, from the running family } r \text { to the searching family } s \text { (or } \\
\text { directly to garage } g \text { without cruising) }\end{array}$ \\
\hline$l_{x, i}$ & $\mathrm{~km}$ & Average distance travelled by car family $x$ in $i$ \\
\hline$\xi_{i}^{m, t}(t)$ & pers/veh & Average passenger occupancy on mode $m$ \\
\hline$N_{o s, i}(t)$ & spots & Total amount of occupied on-street parking spaces \\
\hline$A_{i}$ & spots & Total amount of on-street parking spaces (constant) \\
\hline$\varphi_{i}(t)$ & $\%$ & Probability of finding an available on-street parking space in $i$ \\
\hline$L_{i}(t)$ & $\mathrm{km}$ & Average cruising distance before finding an available on-street parking space in $i$ \\
\hline$T_{c r u, i}(t)$ & $\min$ & Average time spent on cruising \\
\hline$p_{o s}(t) \mid p_{g}(t)$ & $\$ / h$ & Pricing rates for using on-street parking (or garage parking) \\
\hline
\end{tabular}

competitive strategies are analyzed where on-street and garage-parking pricing maximize the same or different objective functions, respectively. Section 4 demonstrates the rationale of the modeling approach with a case study of a typical urban city. System performances under different parking pricing strategies and parking storage capacities are also evaluated. A discussion on parking management policies is provided at the end of the paper.

\section{Methodological framework}

In this section, we present a macroscopic approach for modeling traffic dynamics of multimodal systems with limited parking. For readability, a nomenclature is provided in Table 1 introducing the main variables and parameters utilized in the later text.

\subsection{Multimodal macroscopic traffic model}

Recent findings on the Macroscopic Fundamental Diagram (MFD) significantly advance network-level modeling of urban congestion. The idea of macroscopic traffic model for car-only urban networks was initially proposed by Godfrey (1969). The existence of the MFD with dynamic features and from field data was firstly reported in Geroliminis and Daganzo (2008), showing that urban single-mode regions that are homogeneously congested exhibit an MFD relating the production of a road network (veh-km travelled per unit time) to the network's traffic density. The MFD can be utilized to monitor the performance of road network, requiring data that can be obtained from the existing information technologies from limited measurement points of the network (Ortigosa et al., 2014). An interested reader could refer to Yildirimoglu and Geroliminis (2014) and Leclercq et al. (2014) for a comprehensive review of the recent development on MFD-related theories. Latest works extend the MFD from single-mode application to bi-modal, where cars and buses share the same infrastructure and look at passenger flow dynamics in addition to vehicular dynamics (Zheng and Geroliminis (2013), Geroliminis et al. (2014), Chiabaut et al. (2014) and Chiabaut (2015)). The existence of a mixed-traffic MFD and a three-dimensional MFD (3D-MFD) are investigated in these works via micro-simulation studies. Remarkably, the extended versions of the MFD relate the accumulation (equivalent to traffic density) of cars and buses to vehicular and passenger flows, reflecting congestion dynamics under different mode compositions. Note that we assume in this paper a given production-accumulation MFD, which relates the total distance travelled in region $i$ of mode $m$ (where $m$ can be any vehicular mode that is either utilizing dedicated road space or sharing space with other modes), the production $P_{i}^{m}(t)$, to the vehicle accumulations $N_{i}^{m}(t)$. Mathematically, it can be written as follows for single-mode traffic (e.g. dedicated lanes separating 
cars and buses) and mixed traffic:

$$
P_{i}^{m}(t)=G_{i}^{m}\left(\overrightarrow{N_{i}^{m}}(t)\right)
$$

where $\overrightarrow{N_{i}^{m}}$ is a vector representing accumulation of all modes utilizing the specific road infrastructure; $G_{i}^{m}$ is the MFD function which can be obtained via analytical approximations proposed in Geroliminis and Boyaci (2012) and Leclercq and Geroliminis (2013) or observed with real data from multiple sensors (Leclercq et al., 2014). Under steady-state condition, the outflow (trip completion rate) of a network $O_{i}^{m}(t)$ can be approximated from the production by the following relationship: $O_{i}^{m}(t)=P_{i}^{m}(t) / l_{i}^{m}$ where $l_{i}^{m}$ is the average distance travelled by mode $m$ within region $i$ (note that a trip may cross several regions, $l_{i}^{m}$ represents the distance traveled in $i$ ). We can also obtain the space-mean travelling speed, $v_{i}^{m}(t)$, using its definition, $v_{i}^{m}(t)=P_{i}^{m}(t) / N_{i}^{m}(t)$. The speed is also required later to estimate the travel time of travelling with mode $m$, which influences mode choice of the demand.

\subsection{Parking model}

In this paper we consider a network that contains two typical parking facilities: on-street parking and garage parking. While we focus on the dynamics of a two-facility system, networks with multiple parking facilities can be integrated in our approach, given the choices of the car-users on facility and more detailed origin-destination information.

The on-street parking has a total parking spatial capacity of $A_{i}$, and the occupied parking space at time $t$ is denoted by $N_{o s, i}(t)$ (units of both variables are number of parking spots). The available parking space is thus $A_{i}-N_{o s, i}(t) \geq 0$. We assume that the garage has infinite parking capacity and requires no cruising at the street level (detailed cruising inside a garage is not considered here, as it makes no significant difference for modeling the congestion at street level). Considering limited capacity in the parking garage is investigated in the last section of the paper.

To reflect the impact of parking limitation on traffic, a parking model is introduced. A bi-modal MFD model with unlimited parking such as the one in Zheng and Geroliminis (2013) considers two families of car movements: cars moving towards internal destination and toward external destinations. We now extend the treatment to three families: (I) Cars running toward internal destination but not yet require to search for parking $N_{r, i}(t)$, (II) cars reaching destination and searching for on-street parking space $N_{s, i}(t)$ and (III) cars moving toward external destination regions $N_{o, i}(t)$. Note that cars of $N_{r, i}(t)$ that choose garage parking will be moved out of the system and become outflow of the region without cruising, and we do not set up another specific family for this flow group. Cars that reach destination and successfully park are denoted by $N_{o s, i}(t)$ and $N_{g, i}(t)$ for on-street parking and garage parking, respectively. The interactions among the families will be illustrated later in Fig. 2, when the flow dynamics are introduced. For simplicity, mode index $c$ (for cars) is skipped in these family notations as buses do not search for parking.

For family (II), the cruising-for-parking process is considered to be repetitive Bernoulli trials, which are expressed by a geometric distribution (number of trials until the first success); cruising will continue until an available parking spot is obtained. The probability that a parking spot is available when being reached, labeled by $\varphi_{i}(t)$, is equal to the ratio between the amount of the available space and the total parking space $A_{i}, \varphi_{i}(t)=\left(A_{i}-N_{o s, i}(t)\right) / A_{i}$.

The parking spaces are assumed to be evenly distributed around the destinations, with a spacing of $s_{i}$ between two adjacent spots. Alternatively one may apply a two-dimension cruising model with parking location, provided with detailed data on spatial distribution of parking spaces and the cruising behavior of cars. This is beyond the scope of this work and is discussed in the conclusions of the paper. Given the property of the geometric distribution, the mean of the number of parking spaces passed by a car in family (II) before the car finds an available parking space is, $1 / \varphi_{i}(t)$. The average cruising distance $L_{i}(t)$ thus can be obtained as $s_{i} / \varphi_{i}(t)$, given the spacing parameter $s_{i}$.

Knowing the average travelling speed $v_{i}^{c}(t)$ obtained from the MFD, the cruising delay $T_{c r u, i}(t)$ can be approximated using the equation below:

$$
T_{c r u, i}(t)=\frac{L_{i}(t)}{v_{i}^{c}(t)}=\frac{s_{i}}{\varphi_{i}(t) \cdot v_{i}^{c}(t)}
$$

A more accurate estimation under time-varying conditions is the horizontal difference between the cumulative inflow and outflow time series ( $N$-curves) of the searching state, which is shown in the case study section. Nevertheless, such an estimation is more tedious and it makes quite complex the implementation of a feedback pricing strategy, which is based on current but not predicted information and developed in the next section. Regarding the pricing rates of using the two parking facilities, denote $p_{o s}(t)$ and $p_{g}(t)$ for on-street parking and garage parking when enter the parking facilities at time $t$. Cruising inside garages is neglected.

We shall point out that similar models for treating parking limitation do exist. One model can be found in Geroliminis (2009, 2015), where a parking model was built into a single-mode MFD modeling framework to develop a perimeter flow control strategy for reducing parking cost and total cost of travelers. Another model was developed in Arnott and Inci (2010), where equilibrium conditions under parking constraint (space, duration) are derived for a single-region single-mode and single-parkingfacility system under steady-states. The parking module proposed in this paper is inspired by these literatures, while large amounts of effort are given on integrating such type of model into a multimodal modeling framework so that parking management strategies can be designed and tested for influencing multimodal mobility. Similar state description with simplified cruising dynamics has been developed in Cao and Menendez (2015b). The authors also investigate transition probabilities from different states of the system (cruising, moving) with analytical tools. 


\subsection{System dynamics with MFD representation and parking}

To apply the model, a large-scale city network may require firstly to be clustered into regions that have different characteristics. The criteria for the clustering are: (i) Homogeneous distribution of congestion within each region to obtain a low-scatter MFD (see Ji and Geroliminis (2012) for more details), (ii) a high degree of connectivity and compactness for each region, and (iii) each region operates a single type of mode usage, e.g. mixed traffic or dedicated road spaces everywhere in the region (ongoing work investigates further the last two criteria (Saeedmanesh and Geroliminis, 2015)). Given accumulation $N_{i}^{m}(t)$, we can estimate the outflow of vehicles $O_{i}^{m}(t)$, and of persons $O P_{i}^{m}(t)=O_{i}^{m}(t) \cdot \xi_{i}^{m}(t)$, provided the average passenger occupancy of mode $m, \xi_{i}^{m}(t)$. Given the traffic demand generated at time $t$ of $m, Q_{i}^{k m}(t)$, the flow dynamics of each region $i$ can be described by the change of $N_{i}^{m}(t)$ for vehicle flow, and the change of $N P_{i}^{m}(t)$ for passenger flow. A discrete version of the dynamics can be formulated as flow conservation equations (details for different $m$ is given later).

$$
N_{i}^{m}(t+1)=N_{i}^{m}(t)+\frac{Q_{i}^{k m}(t)}{\xi_{i}^{m}(t)}+\sum_{k} \sum_{j \neq i} O_{j \rightarrow i}^{k m}(t)-\sum_{k} \sum_{j \neq i} O_{i \rightarrow j}^{k m}(t)
$$

In (3), demand $Q_{i}^{k m}(t)$ is the total demand originated from region $i$ (in passengers per unit time), with internal or external destinations $k$ and is divided by the vehicle occupancy (in passengers per vehicle of type $m$ ). The 3rd term of the RHS in (3) denotes the total incoming vehicle flow to $i$ from the adjacent regions $j$ with final destination $k$, while the 4 th term is the outflow to all adjacent regions. $I P_{i}^{m}(t)$ is the incoming flow in person units. Flow conservation for passengers $N P_{i}^{m}(t)$ will have a similar form. The trip length (travelling distance) of vehicles in region $i, l_{i}^{m}$ is considered independent of the destination, thus the transfer flow is $O_{i \rightarrow j}^{k m}(t)=N_{i}^{k m} \cdot O_{i}^{m}(t) / N_{i}^{m}$. High accumulations in the receiving regions might further constrain the transferring flow. Ramezani et al. (2015) utilize a boundary capacity constraint, which rarely occurs when smart traffic management is present. For more info the reader is referred to the above reference. A recent work to consider different trip lengths per destination has been investigated for single regions in Leclercq et al. (2015).

Note that (3) requires a regional route choice model to be integrated between origin-destination pairs travelling by car $Q_{i}^{k c}(t)$. This modeling framework should determine the sequence of the passing regions while moving from $i$ to $k$. Recently such a modeling framework has been developed for car-only multi-region networks (Yildirimoglu and Geroliminis, 2014; Yildirimoglu et al., 2015). While the authors believe that integration of route choice in the current framework is feasible, this is avoided. The main reason is that the route choice involves an iterative procedure to identify region-based equilibrium conditions, which is computationally difficult to be integrated in a pricing optimization. Thus, the sequence of regions for specific ODs is considered known priori. Yildirimoglu and Geroliminis (2014) and Mahmassani et al. (2013) showed that route choice adaptation is more important under congested conditions. While the pricing strategies that are presented here, are able to avoid such conditions, not integrating an assignment framework is not expected to significantly alter the result, with an important computational benefit in parallel. The case study described later in the paper involves an example with two regions that ease the dynamics and route choice description, but still allows to investigate the performance of the pricing strategies under high demand conditions.

To estimate the cruising time for cars, we decompose $N_{i}^{c}(t)$ in Eq. (3) into the three movement families that were introduced in the previous subsection: $N_{i}^{c}(t)=N_{r, i}(t)+N_{s, i}(t)+N_{o, i}(t)$. Buses are assumed to have one family of vehicles with external destinations and no generated "demand", as buses usually run circular routes across the regions with small time-varying service frequencies. Eq. (3) without the demand terms $Q$ is sufficient for describing the dynamics of buses. Fig. 2 displays flow movements of buses and cars with parking choices in region $i$, with all state variables included. We consider parking limitations for only one region, but it can be easily extended to multiple regions. One additional complexity might be that multiple regions with parking limitations would require the addition of a location choice model, with additional cost components (accessibility, distance from the destination etc.). We expect that a simpler formulation eases the physical interpretation of the results.

Assume $\xi_{i}^{c}(t)=1$ for all $t$, the flow conservation of the families I, II, III, and the two families of the parked cars can be written as follows in Eq. 4(a-e) and illustrated with arrows in Fig. 2:

$$
\begin{aligned}
& N_{r, i}(t+1)=N_{r, i}(t)+Q_{i}^{i c}(t)+I_{i}^{i c}(t)-O_{r, i}(t) \\
& N_{s, i}(t+1)=N_{s, i}(t)+O_{r \rightarrow s, i}(t)-O_{s, i}(t) \\
& N_{o, i}(t+1)=N_{o, i}(t)+Q_{i}^{k c}(t)+I_{i}^{k c}(t)-O_{o, i}(t) \\
& N_{o s, i}(t+1)=N_{o s, i}(t)-Q_{i, o s}^{c}(t)+O_{s, i}(t) \\
& N_{g, i}(t+1)=N_{g, i}(t)-Q_{i, g}^{c}(t)+O_{r \rightarrow g, i}(t)
\end{aligned}
$$

In Eq. (4a), $Q_{i}^{i c}(t)$ is the demand traveling internally from region ( $i$ to $i$ ); $I_{i}^{i c}$ is the total incoming flow of vehicles that end their trips in region $i$, where $I_{i}^{i c}=\sum_{j \neq i} O_{j \rightarrow i}^{i c}(t)$. In Eq. (4b), $O_{r \rightarrow s, i}(t)$ is the transfer flow from running (family (I)) to searching-for-parking (family (II)), $O_{r \rightarrow s, i}(t)=O_{r, i}(t) \cdot \omega_{o s, i}^{c}(t)$, where $\omega_{o s, i}^{c}(t)$ denotes the percentage of trip-finishing cars that 


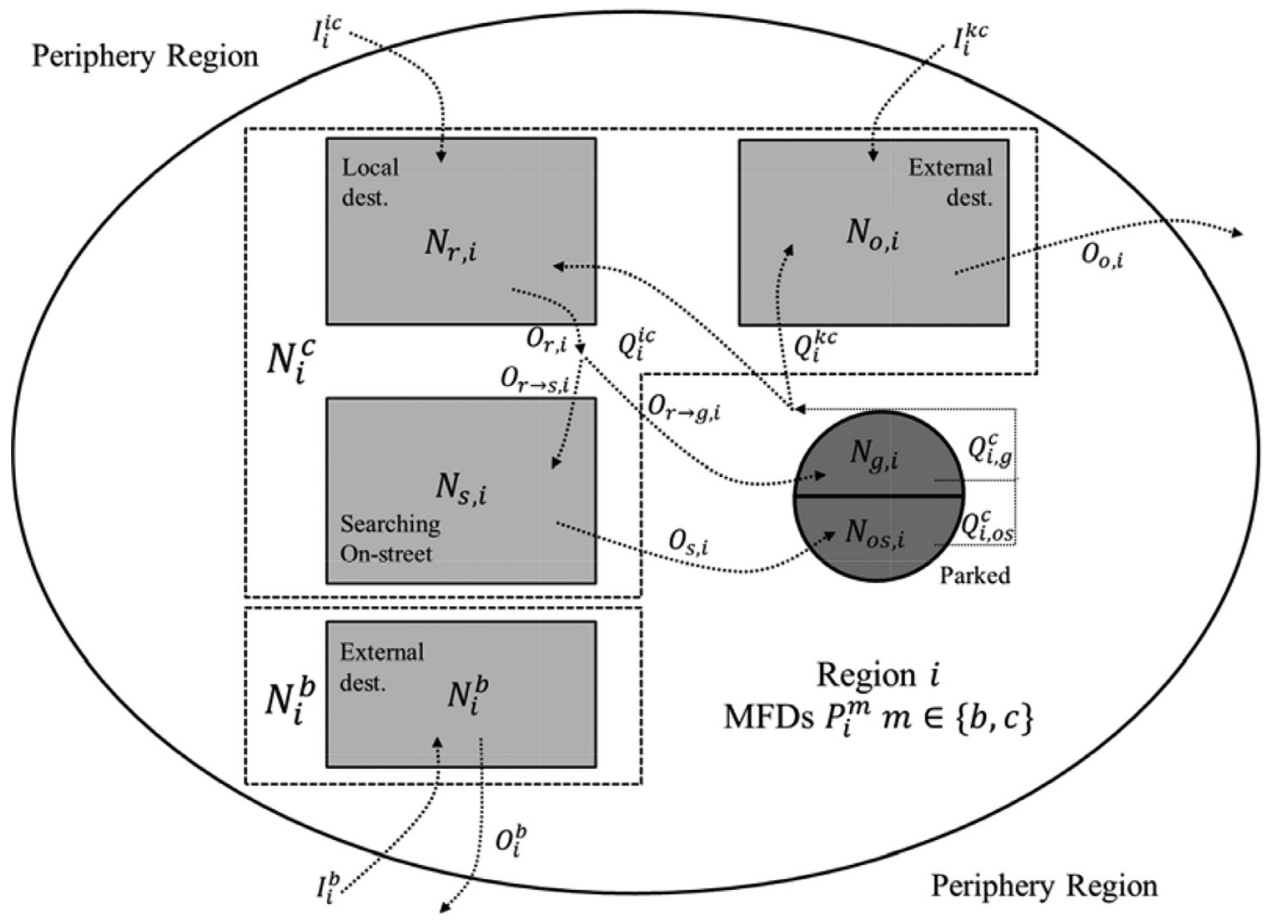

Fig. 2. Illustration of the flow movements in region network $\boldsymbol{i}$ with parking choices, and the interaction with its periphery (adjacent) region.

pursue an on-street parking. The term $Q_{i}^{k c}(t), Q_{i}^{k c}(t)=\sum_{k \neq i} \sum_{j \neq i} Q_{i \rightarrow j}^{k m}(t)$, in Eq. (4c) denotes the demand with external destinations indexed by $k ; I_{i}^{k c}(t)=\sum_{k \neq i} \sum_{j \neq i} O_{j \rightarrow i}^{k m}(t)$ is the total transfer flow traveling through $i$; while the last term $O_{o, i}(t)$ where $O_{o, i}(t)=\sum_{k \neq i} \sum_{j \neq i} O_{i \rightarrow j}^{k m}(t)$, represents the outflow of external destinations. Eqs. (4d) and (4e) describe the dynamics of the parking flows. $Q_{i, o s}^{c}(t)$ and $Q_{i, g}^{c}(t)$ are the demands generated from on-street and garage parking, respectively. The sum of these two terms corresponds to the total demand in Eqs. (4a) and (4c), $Q_{i, o s}^{c}(t)+Q_{i, g}^{c}(t)=Q_{i}^{i c}(t)+Q_{i}^{k c}(t)=Q_{i}^{c}(t)$. The split of the demand between the parking facilities is derived in the next subsection through a mode choice model.

The family-specific outflow $O_{x, i}(t), x \in\{m, g, s, o\}$ can be obtained considering that trip length varies among the different families:

$$
O_{x, i}(t)=\frac{P_{x, i}(t)}{l_{x, i}(t)}=\frac{N_{x, i}(t)}{N_{i}^{c}(t)} \cdot \frac{P_{i}^{c}(t)}{l_{x, i}(t)}
$$

where $N_{i}^{c}(t)=\sum_{x} N_{x, i}(t)$, and $P_{i}^{c}(t)=G_{i}^{c}\left(N_{i}^{c}(t)\right)$ is estimated by Eq. (1). Variable $l_{x, i}(t)$ is the average distance travelled by family $x$ in region $i$ at interval $t$. The family-specific trip distances $l_{m, i}, l_{g, i}$ and $l_{o, i}$ are considered equal, $l_{m, i}=l_{g, i}=l_{o, i}$ and we simply denote them as $l_{c}$. The required cruising distance $l_{s, i}(t)=L_{i}(t)$ is estimated as $s_{i} / \varphi_{i}(t)$, refer to Section 2.2.

\subsection{Aggregated mode choice}

To determine the mode split of the newly-generated demand (between car and bus), a Nested-Logit model (see reference Ben-Akiva and Bierlaire (1999) for a structure of a general Nested-logit model) is applied based on the latest known trip disutility (cost) $C_{i}^{c, f}(t)$ for travelling with cars choosing parking facility from the set $f=$ \{on street versus garage $\}$, and $C_{i}^{b}(t)$ for traveling with buses. For cars, trip disutility includes travel time, cruising delay and the price of parking. While for buses, trip cost consists of travel time, accessing time and the discomfort of on-board overcrowding (see in detail in Zheng and Geroliminis, 2013). The nest is required by the parking facility choice $f$. Denote $\omega_{g, i}^{c}(t), \omega_{o s, i}^{c}(t)$, the percentage of mode choice of cars with garage or on-street parking; and $\omega_{i}^{c}(t)$ and $\omega_{i}^{b}(t)$, the percentage of mode choice of cars and buses. We assume the travelers decide their mode of transport, either car or bus, in the beginning of their trips. Given the current growth of monitoring techniques and the user information systems, it is expected that travelers can have knowledge of associated costs and make smart mode choices (including parking facility choice). The travelers are frequent users of the system, thus they can develop such a knowledge with time, even if less accurate information is available (with a higher uncertainty though), as expressed by higher values in the scale parameters presented in the next paragraph. 
The estimation of bus share $\omega_{i}^{b}(t)$ is through a nested logic structure and given in Eq. (6) below:

$$
\omega_{i}^{b}(t)=\frac{\exp \left(\tau_{b} \cdot C_{i}^{b}(t)\right)}{\exp \left(\tau_{c} \cdot C_{i}^{b}(t)\right)+\exp \left(\tau_{c} \cdot C_{i}^{c}(t)\right)}
$$

where $C_{i}^{c}(t)=\frac{1}{\beta} \cdot \ln \sum_{n} \exp \left(\beta \cdot C_{i}^{c, n}(t)\right)$. Scale parameters $\beta, \tau_{b}, \tau_{c}$ are calibrated to avoid unrealistic oscillation in mode shift. By definition, $\omega_{i}^{c}(t)=1-\omega_{i}^{b}(t)$. Given the total demand $Q_{i}(t), Q_{i}^{m}(t)$ can be estimated by $\omega_{i}^{m}(t)$. Given the car demand generation $Q_{i}^{c}(t), Q_{i, o s}^{c}(t)$ and $Q_{i, g}^{c}(t)$ in Eq. (4) can be obtained.

Note that the car-users have limited knowledge on the possible availability of the on-street parking by the time they start their trip. Then if they chose to travel by car, when they get in the proximity of the destination, they re-evaluate the costs of on-street and garage parking (e.g. via parking guidance system), and they make a new decision. If they decide to travel by bus, they do not have such an option. Therefore the final choice on parking facility is determined on-site. While the a-priori and the final decision are the same in the majority of the case, we consider such a framework more realistic. If car travelers remain in the initial decision of on-street parking, higher cruising times (or even gridlock situations) might be created, especially when on-street parking becomes full during their trip. The distribution of the arriving flow $O_{r, i}(t)$ between the two parking facilities is estimated with a Logit model by percentages $\omega_{g, i}^{c}(t)$ and $\omega_{o s, i}^{c}(t)$, where $\omega_{o s, i}^{c}(t)=\exp \left(\beta \cdot C_{i}^{c, o s}(t)\right) /\left\{\exp \left(\beta \cdot C_{i}^{c, o s}(t)\right)+\exp \left(\beta \cdot C_{i}^{c, g}(t)\right)\right\}$. The production of $O_{r, i}(t) \cdot \omega_{o s, i}^{c}(t)$ thus is the input to the cruising family $N_{s, i}(t)$. The developed mode choice model contains a small number of parameters that can be estimated with standard procedures (Ben-Akiva and Bierlaire, 1999) once data is available. This should be a research priority.

\section{Parking pricing strategies}

Road traffic networks and management systems constitute a system of systems (SoS) with complex behaviors of various modes of transport competing for the same space. A common approach of decentralized control design for traffic management is not appropriate for heterogeneously loaded networks with multiple modes of transport, short links and spillbacks, resulting in the saturation of neighboring networks (Haddad et al., 2013). Lack of collaboration between operators of different systems (e.g. traffic signals, parking facilities, public transportation) can result in sub-optimal (or even worse conditions compared to donothing) because it does not consider interactions and conflicts between different networks and transport modes. The modeling of the previous section allows for the investigation of holistic traffic management schemes based on pricing of on-street and garage parking. The historical administrative organization of metropolitan areas has led road systems to be operated by a patchwork of different systems without proper level of communication in exchange data and actions. Most importantly, they are often competing with one another to avoid congestions on their respective networks. These systems and operators are rather closed with little information shared between them, both for technical reasons and lack of motivation to share strategic information. Traffic systems, being inherently a distributed and interconnected process, should be managed as a whole to improve their global operational efficiency while avoiding conflicting decisions. MFD-based traffic management strategies serve for this sustainable and global mobility goal. Recent studies have demonstrated the performance of such strategies, examples including congestion pricing (Zheng et al, 2012; Simoni et al., 2015), space allocation for bus lanes (Zheng and Geroliminis, 2013), and dynamic traffic signal perimeter control (refer to Ramezani et al., 2015 for a review).

We investigate now an MFD-based parking management, with two dynamic pricing strategies P1 and P2, to determine onstreet parking pricing rate $p_{o s}(t)$ and garage pricing rate $p_{g}(t)$ such that the congestion of traffic and cruising-for-parking are reduced. We assume to have full authority of both prices whereas in reality they belong to parties with different operating objectives (and competition might occur). Therefore we further introduce an optimization procedure to determine the prices when competition exists between on-street parking and garage parking operators. Pricing is only applied in the center region which suffers more congestion, note that a region index is omitted from the above pricing variables. Strategy P1 considers that the dynamic evolution of the system is perfectly known and solves a single full-horizon optimization problem to minimize the total costs experienced by all passengers. While such an approach can provide close to system optimum conditions, it is also difficult to implement due to lack of accurate prediction and high computational effort to solve a highly non-linear problem. Nevertheless, it gives an upper limit for comparison purposes with more feasible strategies like a feedback based P2 strategy.

Strategy P2 develops a generic control-loop feedback mechanism. It tries to succeed two set points, related to (i) the maximum production of the network (in terms of vehicle-kilometers travelled per time interval) and (ii) small cruising time for on-street parking. This strategy calculates an error value as the difference between a measured process variable and the desired set point. It attempts to minimize the error by adjusting the prices $p_{o s}(t)$ and $p_{g}(t)$.

Then, strategy competition describes the competition behavior between the two parking operators, where one operator reacts to the decision of the other operator and determines the best pricing rates to maximize each objective with certain tolerance. This interactive process is assumed to last until, if exists, a convergence reached between the two competitors.

\subsection{Strategy P1: A system optimum parking pricing scheme}

This strategy aims at achieving a system optimum. The goal of this pricing scheme is to minimize the total passenger cost (TPC), including travel time, cruising time and parking fees, and serve the total network demand. The control variables are parking 
prices in the center region controlled by a central operator with a full knowledge of the system. Such a strategy is difficult to be implemented due to prediction limitations and day to day variations of demand. Mathematically it can be described as the following in Eq. (7):

$$
\min _{p_{o s}(t), p_{g}(t)} Z_{P 1-A}=\sum_{t, i, m} \operatorname{TPC}_{i}^{m}(t)=\sum_{t, i, m}\left(\operatorname{PHT}_{i}^{m}(t)+\operatorname{Tos}_{i}^{c}(t)+\operatorname{Tg}_{i}^{c}(t)\right)
$$

where the total passenger hours travelled (PHT) can be easily estimated given the accumulations in Eq. (3). $\operatorname{Tos}_{i}^{c}$ and $T g_{i}^{c}$ are the total parking tolls of on-street parking and garage parking, respectively. Both terms can be calculated given the pricing rates $p_{o s}(t)$ and $p_{g}(t)$, and the number of users by Eq. (5). Let us refer (Eq. (7)) as P1-A.

We also introduce a P1-B strategy, as written in Eq. (8), where the prices are determined only to optimize the total travel time.

$$
\min _{p_{o s}(t), p_{g}(t)} Z_{P 1-B}=\sum_{t, i, m} \operatorname{PHT}_{i}^{m}(t)
$$

We will compare and discuss later the efficiency of the two schemes. Traditionally from an economic prospective, pricing is considered a transfer of money from one entity to another (e.g. from car travelers to system operators) and this amount could be utilized to improve the service of more sustainable modes, e.g. like public transport. While the literature review of pricing is vast, pricing implementations are limited and this is because people are reluctant to pricing and user acceptability is questionable. In this work we are interested in considering strategies, where the amount of toll paid is included in the optimization, so that these strategies become more attractive and have a more socially-oriented objective. Results will highlight some interesting properties of such integration.

Problems (7) and (8) are subject to the system dynamics introduced in the previous section. We also add a constraint that $p_{o s}(t)<p_{g}(t)$. The reason is the following: Since on-street parking requires cruising time and garage capacity is considered infinite, without imposing this constraint, the cost of garage parking would be always less and the optimization will consequently rule out the option of on-street parking. The optimization problems (7) and (8) are highly non-linear. We solve these problems by sequential quadratic programming (Nocedal and Wright, 2006). We apply this algorithm for multiple initial values (around 1000) to avoid convergence to local minima, which might be the case for a non-smooth objective function.

\subsection{Strategy P2: A congestion- and cruising-responsive feedback parking pricing scheme}

This pricing scheme is designed to reduce both traffic congestion caused by cars in general and by the cruising traffic. The linear control theory offers a number of methods and theoretical results for feedback regulator design in a systematic and efficient way. Feedback type controllers of PID type have been widely applied in freeway traffic flow management, e.g. Papageorgiou and Kotsialos (2002) as the core control law to control flow entering from on-ramp to main freeway. Stability analysis has shown the global convergence of the controllers: "For all choices of parameters, the strategy preserves closed-loop stability and forces the actual control variables to reach the desired one" (Kosmatopoulos and Papageorgiou, 2003). Recently, similar strategies have been introduced for MFD perimeter control for multiple regions (Aboudolas and Geroliminis, 2013 ; Keyvan-Ekbatani et al., 2015; Haddad, 2015). The purpose of these strategies is to retain accumulation of vehicles at a desired level that maximizes system outflow, without the direct need for demand information or prediction. The regulators are strictly based on real-time measurements, without any need for online model or demand predictions. A state feedback controller based on occupancy information has been recently introduced for multiple parking location management, without treating cruising-for-parking (Qian and Rajagopal, 2014). Nevertheless, for the problem formulation of the previous section, controlling only the accumulation of cars will not resolve the problem of congestion as cruising-for-parking significantly contributes as well. Here we intend to apply the same principle as in urban traffic management, where the difference (and the major difficulty) come from the facts that (A) pricing affect user's choice, whereas in the ramp-metering or network-level entry metering case users have to obey the controlled traffic signals, and (B) the controller that determines the dynamic pricing for influencing mode choice and parking facility choice, aims to achieve two control objectives simultaneously which experience correlation. Point (B) is challenging and innovative, given that such control scheme is rarely reported in the traffic management literature. We will demonstrate later that this controller functions effectively in reducing both the general congestion and cruising delay.

To provide an overview of the developed feedback control scheme, Fig. 3 shows a block diagram of the control logic. The control problem is to regulate the number of vehicles cruising for parking and the total number of vehicles moving in the city center to maintain them around selected set-points (indicated by the red arrows in Fig. 3), respectively, via appropriate manipulation of the prices of on-street and garage parking, $p_{o s}$ and $p_{g}$. The prices will influence mode choice in the beginning of the trip, where the demand of the center region, $Q_{i}^{i}$, is split into $Q_{i}^{i c}$ and $Q_{i}^{i b}$ (same applies for the demand from periphery regions with center region $i$ as destination, as travel cost of all passing regions are taken into account). They will also influence the on-site parking facility choice, when the outflow from $N_{m}$ will become either part of $N_{s}$ searching for on-street parking spot or directly parking in the garage. Please note that although the enforcement of pricing strategies will affect the individual choice behavior of users, the collective traffic flow dynamics follow the MFD, the shape of which is not very sensitive to the slow timely-varying behavioral changes. This property allows the MFD to provide reliable information on system performance and to determine the optimal prices in real-time. It becomes more complicated, however, if routing strategies are implemented. This is because routing may influence the trip lengths of users which have direct impact on the MFD (Yildirimoglu and Geroliminis, 2014). In addition, we 


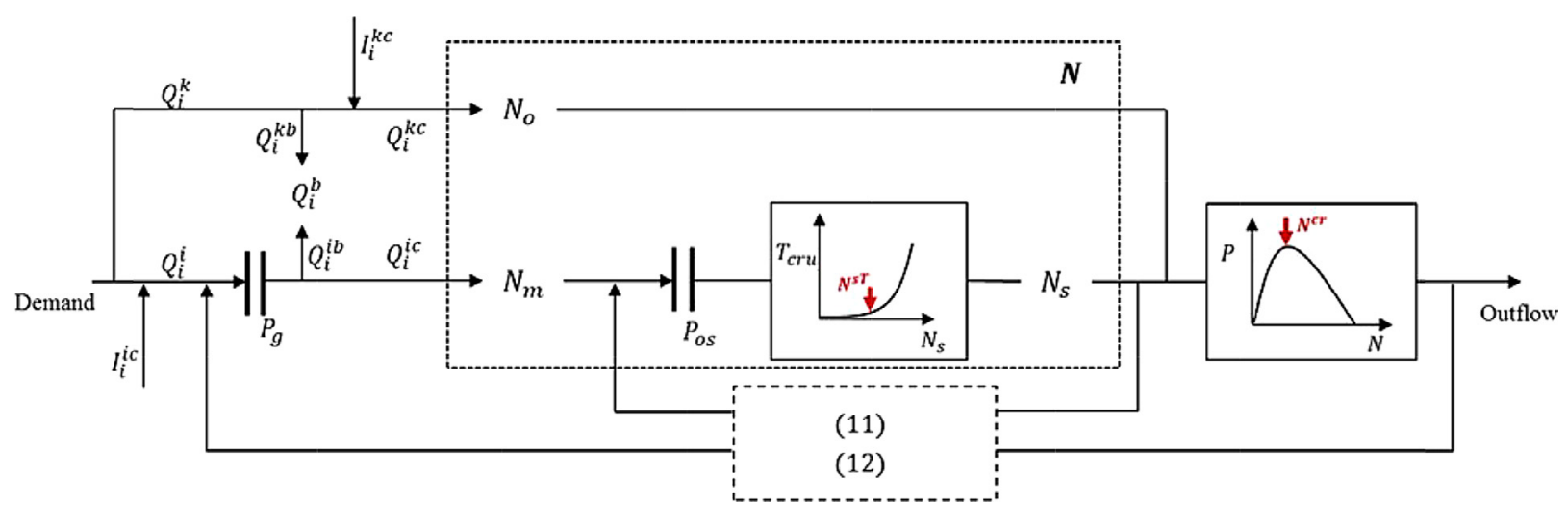

Fig. 3. A block diagram of the proposed pricing controllers.

consider that demand with destination outside of the center region where there is no parking limitation is not affected by pricing as they will not be subject to paying a price, thus can be considered as disturbance to the controlled system (the top part of the block diagram).

Now let us describe the control logic for pricing in more detail. Car users will pay a parking fee based on the magnitude of congestion they cause at the moment they enter the network. A feedback-type I (integrated) controller is employed to update the time-dependent prices $p_{o s}(t)$ and $p_{g}(t)$, where the control variables are the total accumulation $N^{c}(t)$ and the accumulation of cruising family $N_{s, i}(t)$. Eqs. (9) and (10) describe mathematically the two control mechanisms respectively, where $c_{1}$ and $c_{2}$ are control gain parameters:

$$
\begin{aligned}
& p_{g}(t+1)=p_{g}(t)+c_{1}\left(N^{c}(t)-N^{c r}\right) \\
& p_{\text {os }}(t+1)=p_{\text {os }}(t)+c_{1}\left(N^{c}(t)-N^{c r}\right)+c_{2}\left(N_{s}(t)-N^{s T}\right)
\end{aligned}
$$

The concept is that garage users have to pay for the hyper-congestion due to large accumulations of cars in the network, while on-street users have to pay for the cost of cruising as well. In this way, both types of congestion can be eliminated. Eq. (9) states that the garage price for the next time interval, $p_{g}(t+1)$, increases if the accumulation of car users $N^{c}(t)$ exceeds the critical accumulation of the network $N^{c r}$. Recall that $N^{c r}$ is the point in the MFD, where the maximum network production is reached and network production decreases if $N^{c}(t)>N^{c r}$, see in Fig. 1. A slightly smaller value is chosen, e.g. $0.95 N^{c r}$, to facilitate stability of the system around the set point. Eq. (10) states that the on-street price $p_{o s}(t+1)$ charges the same amount for the congestion, and an additional amount for causing cruising delay which is proportional to the difference between $N_{s}(t)$ and a pre-defined threshold $N^{s T}$. Parameter $N^{s T}$ can be interpreted as the tolerated amount of cruising vehicles, which reflects the level of cruising delay. Cruising time is considerably small for a wide range of values of $N_{s}$, the number of cruising vehicles. Thus, $N^{s T}$ is chosen to keep the value of cruising at a reasonably low value (e.g. less than 2 min). Having $c_{1}$ parameter in both (9) and (10) simply means that all car users have to pay for congestion independently of the parking choice. If $c_{2}=0$, then these strategies are similar to the existing ones in the literature, but the large disturbance generated by cruising-for-parking will not allow the system to converge in the desired accumulation threshold. Good values of regulator gain may be found with appropriate Control Engineering methods or manual fine-tuning; feedback regulators are quite robust to moderate parameter value change. Strategy P2, in contrast to P1, does not require any prediction and is based only on quantities that can be estimated with sensing techniques. Our analysis shows that even under perfect information, the performance of P1 in terms of PHT is the lower bound that P2 can reach, when uncertainty is added in the P1 demand and mode choice, P2 outperforms P1, which also has a high computational burden.

Regarding the choice of $N^{c r}$ and $N^{s T}$, they can be seen as policy factors influencing the service level of general mobility and onstreet parking, respectively. One may argue that if lower values are chosen, the toll is very strict and over-charging might occur as the system operates in a state less than its capacity. Nevertheless, this does not necessarily indicate over-charging unless the total toll paid outweighs the total savings in travel time. System operator can choose the desired values in a way to maximize traffic flow and as a result to transfer the highest possible number of passengers with small delays. The value of toll which results in points in the MFD just without the decreasing branch can be set as the lower bound, while the one which results the total toll paid just equal to total travel time savings can be set as the upper bound in order to avoid over-charging. This higher toll will push the system to operate at a smaller than the maximum flow during the toll period, but with higher average speed and some potential savings in travel delay. This also implies that an operator can utilize a slightly smaller value to guarantee a more reliable operation, e.g. capable of buffering if there is sudden disturbance from demand, though fewer users are served, as flow decreases (see for example Geroliminis and Levinson, 2009). 


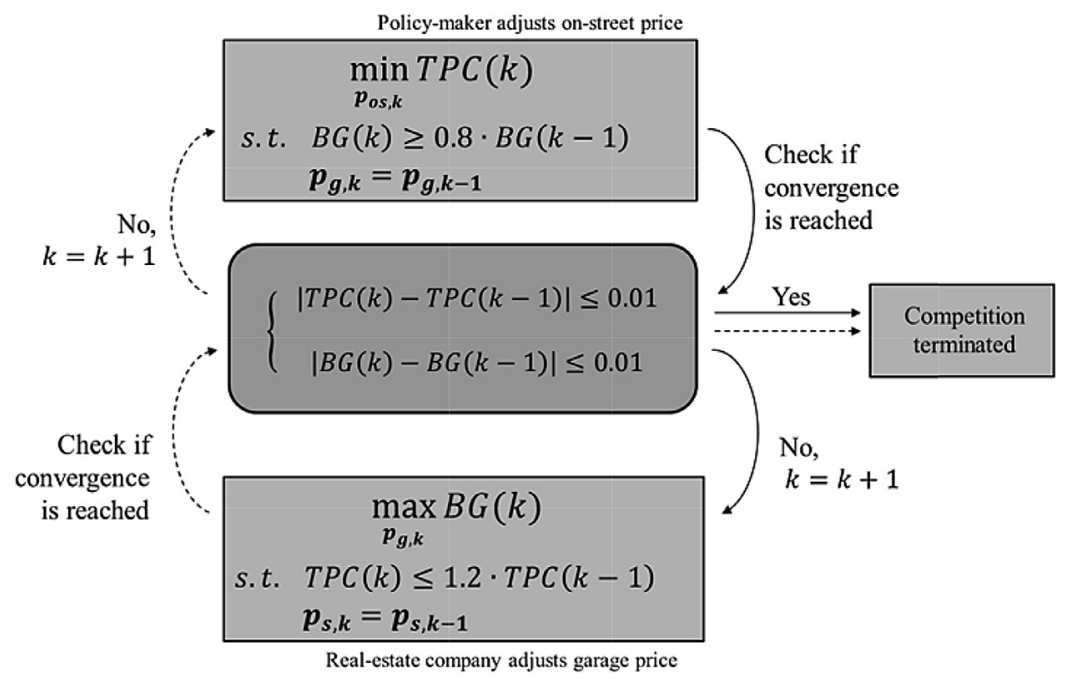

Fig. 4. A graphical illustration of the pricing competition process between the two parking operators.

\subsection{Strategy competition: An interactive two-player pricing competition}

Note that in P1 and P2, we assume that a central decision-maker operates both parking facilities and has full power to determine $p_{o s}(t)$ and $p_{g}(t)$. In practice, the authorities belong to different operators. City policy-makers operate on-street parking while private companies operate garage parking. These operators might have different objectives, e.g. city tries to minimize TPC by controlling the price of on-street parking, while garage operator maximizes its profit, the revenue benefit of garage pricing, denote it BG, by controlling the price of garage parking. Then the optimization problem can be re-formulated as the follows:

$$
\begin{aligned}
& \min _{p_{\text {os }}(t)} \mathrm{TPC}=\sum_{t, i, m}\left(\mathrm{PHT}_{i}^{m}(t)+\operatorname{Tos}_{i}^{c}(t)+\operatorname{Tg}_{i}^{c}(t)\right) \\
& \max _{p_{\text {og }}(t)} \mathrm{BG}=\sum_{t, i} \operatorname{Tg}_{i}^{c}(t)
\end{aligned}
$$

It can be seen that direct conflicts, represented by the term $\operatorname{Tg}_{i}^{c}(t)$ in Eqs. (11a) and (11b), exist between the two operators. This pricing competition can be viewed as a responsive and negotiate-alike process, where each operator changes the pricing strategy after recognizing the impact of the other party's action. In game theory, these types of problems are known as differential games that try to analyze conflicts in dynamical systems. Differential game models have been used extensively to study problems of conflict arising in such diverse disciplines as operations management, marketing, and economics. Of great attention are the Stackelberg differential games (Bagchi, 1984). The leader announces its control path, i.e. on-street pricing for the time horizon of our problem. Then, the follower decides its optimal control path in response to the announced $p_{o s}(t)$. Stackelberg equilibrium is obtained when the leader solves its problem taking into account the optimal response of the follower.

We consider solving the formulated parking competition by a leader-follower optimization procedure, where the policymaker as the higher level leads the process and the companies at the lower level follows. Given the complexity of the optimization problem, we follow an iterative process with an objective to converge to a fixed point with small error. At each competition round, each operator tries to optimize its own objective given the action of the previous one. To avoid oscillatory behavior of the optimization procedure and mimic the acceptance boundary of the parties during negotiation, we add constraints that the actions of the one optimization problem should not worsen the other problem by more than $20 \%$ compared to the previous iteration. A graphical illustration of this process is displayed in Fig. 4. Comparing to the existing applications of bi-level optimization in transport-related researches, such as in Yin (2002), the challenges here are two-fold: (i) The two objective functions have direct conflict, given that $\operatorname{Tg}_{i}^{c}(t)$ is in both objective functions, and (ii) if there exists a fixed point where the two operators cannot improve their profit, i.e. the final values of the both objective functions vary within $1 \%$ of the previous round of the competition. Results are presented in the following section.

\section{Case study and analysis}

The different pricing strategies are tested in a concentric two-region bi-modal network, which is governed by the dynamics as described in Sections 2.1-2.4 by Eqs. (1)-(6). Denote "1" and "2" for the center and the periphery regions, respectively. The 
dynamic Eq. (3) thus can be adapted as follows:

$$
\begin{aligned}
& N_{1}^{1 c}(t+1)=N_{1}^{1 c}(t)+Q_{1}^{1 c}(t+1)+O_{2}^{1 c}(t)-O_{1}^{1 c}(t) \\
& N_{1}^{2 c}(t+1)=N_{1}^{2 c}(t)+Q_{1}^{2 c}(t+1)-O_{1}^{2 c}(t) \\
& N_{2}^{1 c}(t+1)=N_{2}^{1 c}(t)+Q_{2}^{1 c}(t+1)-O_{2}^{1 c}(t) \\
& N_{2}^{2 c}(t+1)=N_{2}^{2 c}(t)+Q_{2}^{2 c}(t+1)+O_{1}^{2 c}(t)-O_{2}^{2 c}(t)
\end{aligned}
$$

Then the regional accumulation can be defined as $N_{1}^{c}(t)=N_{1}^{1 c}(t)+N_{1}^{2 c}(t)$ and $N_{2}^{c}(t)=N_{2}^{1 c}(t)+N_{2}^{2 c}(t)$. Eq. (12a) dictates that the change of the accumulation of the running traffic that generated from region 1 with destination region 1 (internal traffic), is the sum of the demand entering the network (the 2nd term RHS) and the transfer flow entering region 1 from region 2 (the 3rd term RHS), minus the transfer flow (the 4th term RHS) reaching the destination in region 1. While for external traffic, Eq. (12b) captures a similar form with (12a) except that travelers finishing their trip in region 2 and starting travelling in region 1, will all finish in region 1 not returning to region 2 (assuming no cross-region trips). Eqs. (12c) and (12d) can be interpreted as above. While for buses, only two dynamic equations are needed, because in general buses serve across regions over time:

$$
\begin{aligned}
& N_{1}^{b}(t+1)=N_{1}^{2 b}(t+1)=N_{1}^{2 b}(t)+O_{2}^{1 b}(t)-O_{1}^{2 b}(t) \\
& N_{2}^{b}(t+1)=N_{2}^{1 b}(t+1)=N_{2}^{1 b}(t)+O_{1}^{2 b}(t)-O_{2}^{1 b}(t)
\end{aligned}
$$

For passenger flow dynamics, similar laws apply given the passenger occupancy information. The parking dynamics Eqs. (4a)-(4e) remain unchanged, given that only the center region $(i=1)$ has parking limitation.

Mixed traffic of buses and cars occurs in the outside region (periphery), while a fraction of road space in the center region (center) is pre-determined and dedicated to bus usage only. This fraction is estimated by an optimization approach with an objective to minimize system delays where cruising is negligible and pricing is absent (the interested reader could refer to Zheng and Geroliminis, 2013). We simulate an urban road traffic system during a typical morning period for $4 \mathrm{~h}$ (at a 3-min time step, in total 80 time units). ${ }^{1}$ Travel demand has a symmetric trapezoidal shape with time and the length of peak period equal to $1 \mathrm{~h}$. A $70 \%$ fraction of the demand generated in the periphery will travel to the city center and the $70 \%$ fraction of the demand generated in the city center will remain. A mixed bi-modal MFD for the periphery and two single-mode MFDs for the center are estimated with Variational theory based on Boyaci and Geroliminis (2012) and Leclercq and Geroliminis (2013), for a grid network with the following settings: (i) two-lane one-way roads with block lengths $154 \mathrm{~m}$ and $308 \mathrm{~m}$ in the two regions respectively, (ii) signal settings for all intersections, green of $40 \mathrm{~s}$; cycle of $90 \mathrm{~s}$ and offset of $0 \mathrm{~s}$, (iii) a triangular fundamental diagram for each link with free flow speed for buses and cars $15 \mathrm{~m} / \mathrm{s}$; jam density $0.15 \mathrm{veh} / \mathrm{m}$ for cars and $0.05 \mathrm{veh} / \mathrm{m}$ and congested wave speed for buses and cars, $5 \mathrm{~m} / \mathrm{s}$. The functional form of the MFD for the center region: (i) $P_{1}^{c}(t)=0.16 e^{-22.76 \frac{N_{1}^{c}(t)}{\beta \cdot L_{T}}} \cdot N_{1}^{c}(t)$ for $N_{1}^{c}(t)<N^{c r}\left(N^{c r}=0.15 \beta\right.$. $L_{T}$, and else $P_{1}^{c}(t)=0.13 \beta \cdot L_{T}-0.87 N_{1}^{c}(t)$, where $\beta \cdot L_{T}$ is the $\beta$ percentage of the total-lane-km length allocated to the car network; (ii) $P_{1}^{b}(t)=0.16 e^{-72.74 \frac{N_{1}^{b}(t)}{(1-\beta) \cdot L_{T}}}$ for $N_{1}^{b}(t)<N_{b}^{c r}\left(N_{b}^{c r}=0.05(1-\beta) \cdot L_{T}\right)$, and else $P_{1}^{b}(t)=\left(1.52-30.48 \frac{N_{1}^{b}(t)}{(1-\beta) \cdot L_{T}}\right) \frac{N_{1}^{b}(t)}{(1-\beta) \cdot L_{T}}$. We consider two values for $\beta, 95 \%$ for off-peak and $85 \%$ for off-peak hour (thus $\beta(t)$ ), assuming dynamic space allocation strategy is applied. Two modes of transport are considered available in the system, car and bus. We assume that a fraction $10 \%$ of the users are captive bus users and do not have access to cars. The average distance between on-street parking spaces $s_{i}=20 \mathrm{~m}$. The duration of on-street parking is $1 \mathrm{~h}$. A sensitivity analysis on parking duration will be reported in the next section (Section 5.3). The MFD forms are consistent with empirical and simulated findings from Geroliminis and Daganzo (2008) and Geroliminis et al. (2014).

In the subsections below, we show the dynamics of the two-region bi-modal system under parking limitation and pricing, illustrating the mechanism of the developed model. Through Sections 4.1-4.4 we present a performance comparison of five parking pricing policies: A base scenario with free on-street parking, a flat parking-rate scenario for both garage and on-street, Strategy P1 (A and B) and Strategy P2. The base scenario applies a time-constant parking garage fee $p_{g}$, which creates crowding for the cruising traffic. The flat-rate scenario estimates constant values of $p_{o s}$ and $p_{g}$ by minimizing objective function (7). Given these results, the last Section 4.5 utilizes the proposed approach to reproduce the pricing competition dynamics between the parking authorities with different management goals.

\subsection{System dynamics with constant pricing}

Fig. 5 illustrates the system dynamics of the center region (the region index is skipped in the text), under an optimal fixedpricing scheme where the prices are obtained through optimization, minimizing the total cost of the users, see objective function

\footnotetext{
1 The choice of the time step in this paper ( $3 \mathrm{~min}$ ) is consistent with the existing literature based on empirical observations, that show little scatter in the MFD shape, for homogeneously congested regions. Measurement errors (in the range of 10\%) in the MFD function in the dynamic equations, do not influence the performance of pricing.
} 

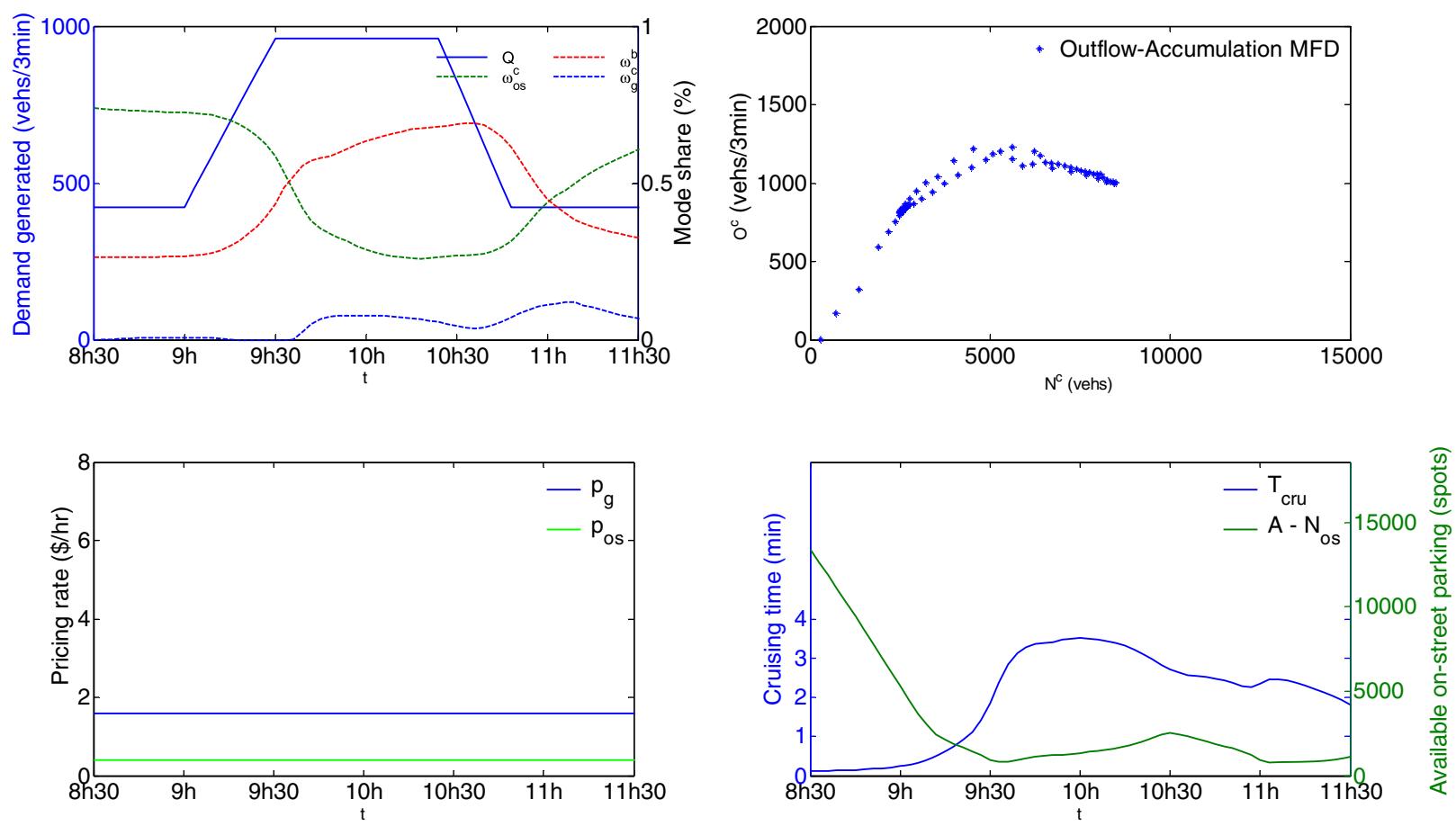

Fig. 5. System dynamics and traffic performance under the optimal constant parking pricing scheme for the center region: (a, top left) mode share of the three modes and total travel demand over time, (b, top right) the MFD of the center region car network with congestion observed, (c, down left) the pricing rates of $p_{\text {os }}$ and $p_{g}$, and (d, down right) average cruising time and the free parking space over time.

of Eq. (9) without a time index for the decision variables. The optimal prices are $p_{o s}=0.4 \$ / \mathrm{h}$ and $p_{g}=1.6 \$ / \mathrm{h}$ (Fig. $5(\mathrm{c})$ ). Fig. 5(a) shows the time series of total demand and the mode share for buses, on-street and garage parking. It can be observed that mode share of buses increases during peak-hour as traveling with car experiences higher travel costs than bus. Garage parking is chosen only after congestion builds up and on-street parking is saturated with high values of cruising (around time 9:30). From the outflow-accumulation MFD in Fig. 5(b) (with the critical accumulation $N^{c r}=5200 v e h$ and a decreasing branch up to $N^{c}(t)=9000$ veh for car network), we can clearly see that the car network experiences congestion. Judging from the time series of cruising delay in Fig. 5(d), cruising-for-parking contributes to the high travel cost of cars as well. Nevertheless, even if demand is high, on-street parking is not fully occupied because travelers have alternatives of lower total cost (e.g. public transport or garage parking with fee). Note that such a static pricing scheme cannot avoid neither congestion nor cruising. The travel cost of buses remains nearly the same since buses operate on the dedicated lanes with scheduled frequencies, although a slight increase can be found in Fig. 5(d) during the peak-hour, which is a reflection of speed reduction due to the longer dwelling time for boarding more passengers. Congested states can be observed in the MFD in Fig. 5(b), where different values of outflow occur for the same accumulation. The reduction of outflow can be explained by the reduction of on-street parking availabilities. As cars have to cruise longer distance to compete for a parking space, the outflow drops accordingly (recall that outflow is production over trip length). This analysis is convincing for the need of more advanced pricing schemes to eliminate cruising and states in the decreasing (congested) part of the MFD.

\subsection{System dynamics with strategy $P 1-A$}

Now let us examine the performance when dynamic pricing strategy P1-A is applied. The goal of this pricing scheme is to minimize the total passenger cost (TPC) shown in Eq. (9), including travel time, cruising time, parking fees controlled by a central operator with a full knowledge of the system. Prices can vary every 15 min ( 5 interval units). The same graphs of Fig. 5 are reproduced and displayed in Fig. 6. Evidently, the cruising delay and accumulation decrease significantly (see Fig. 6(b)). Higher maximum outflow can be observed from the MFD (nearly 1500 in this case, versus 1200 in the fixed pricing case). With a careful investigation on the resultant mode shares of bus, we conclude that the large improvement of performance under strategy P1-A is due to its capability of triggering an earlier mode shift from cars to buses during the on-set of the peak-hour. Remarkably, such small change (roughly a mode share difference of $2 \%$ during $20 \mathrm{~min}$ ) in the mode share creates significantly different traffic performances. Furthermore, it should be highlighted that less toll is charged on the users by strategy P1-A. Nevertheless, during the optimization the travelers' choices and traffic states are perfectly predicted for the whole horizon, which is unrealistic. Imperfect information and lack of accurate modeling of traffic states and user behavior can deteriorate the performance of pricing. 

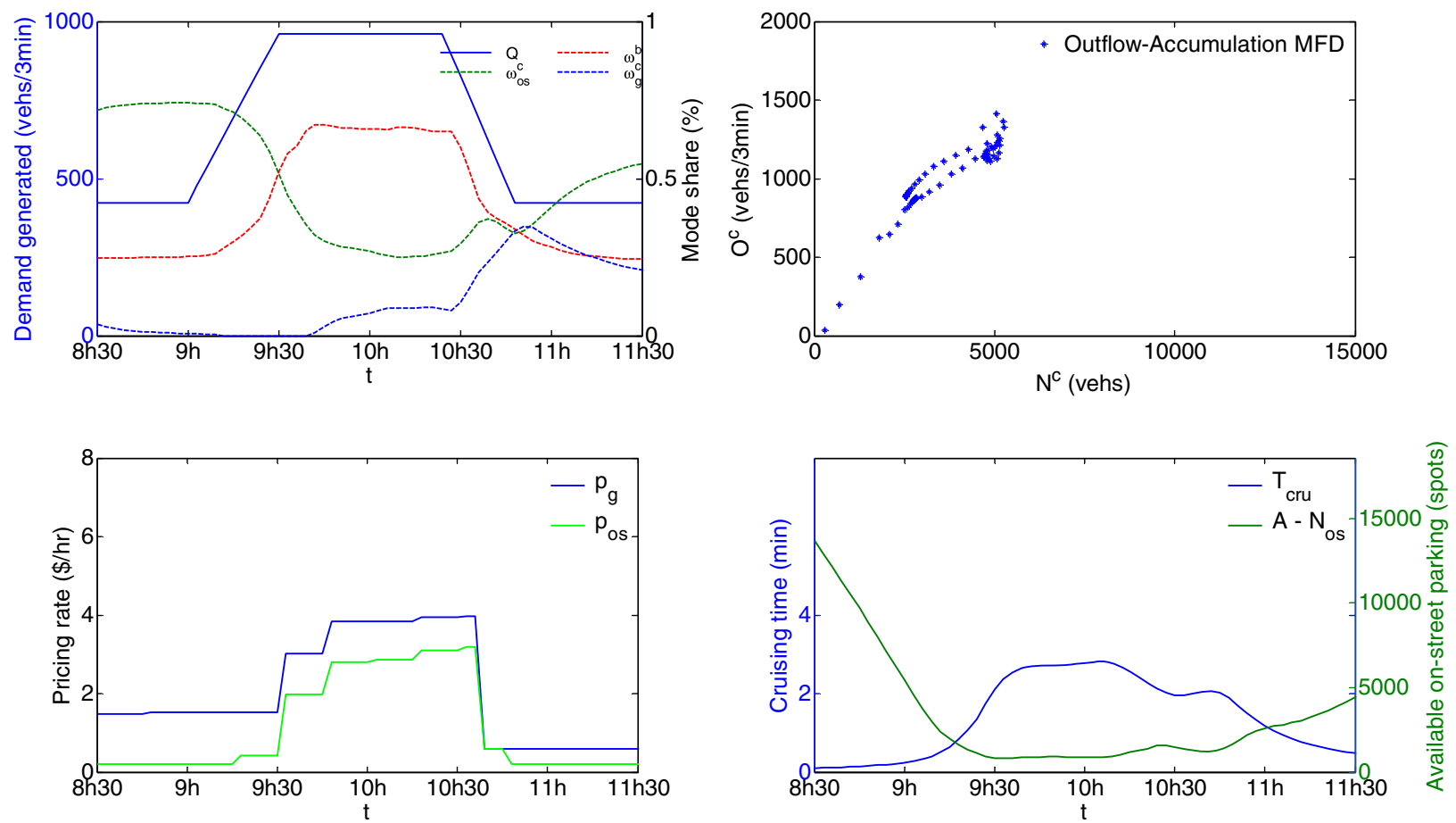

Fig. 6. System dynamics and traffic performance under the optimal strategy P1-A. The same graphs are reproduced as in Fig. 5..

\subsection{System dynamics with strategy $P 2$}

Fig. 7 illustrates the resultant system performance under the feedback-type time-dependent pricing strategy P2. Similarly as P1 strategies, prices are controlled every $15 \mathrm{~min}$. Fig. 7(a) plots the number of cruising vehicles $N_{s}$ versus cruising time $T_{c r u}$ for each time period for the no-pricing case. Note that for a range of $N_{s}$ the cruising time is quite small ( $<1 \mathrm{~min}$ ), that allows the development of an efficient feedback control strategy that eliminates both cruising and congestion. For the applied strategy P2 here, the objective is to control the cruising delay $T_{c r u}$ under $3 \mathrm{~min}$, therefore we choose a value of 900 veh for $N^{s T}$. Regarding $T_{c r u}$, it may be monitored directly from existing data collection technology and used to control the price on cruising. Parkingbehavior-related studies, e.g. Martens et al. (2010) have considered that cruising traffic makes circular movements within certain local blocks around their destinations. Provided with the high-resolution GPS data, trajectories of vehicles can be reproduced. With such information, the cruising-for-parking part of a trip can be distinguished from the normal running part (constructing a model to estimate cruising time then is feasible). Alternatively, a system where the driver requires a shortest path route through existing navigations, could integrate the information of the destination to identify when vehicles start cruising. Fig. 7(d) displays how the accumulations $N^{c}(t)$ and $N_{s}(t)$ fluctuate closely around the critical values $N^{c r}$ and $N^{s T}$, though there are a few cases where congestion exceeds the desired states. This shows consistency of the developed controller with the expected system dynamics, even if the disturbances of the system are non-zero. Fig. 7(b) shows the Outflow-Accumulation MFD pairs during the simulation, with no points in the congested regime. In Fig. 7(c), the time-dependent pricing rates $p_{o s}(t)$ and $p_{g}(t)$ are plotted. The average pricing rates are found slightly higher during the peak hour, compared to Strategy P1-A.

To provide additional insights on how different pricing strategies influence the dynamics of the general and the cruising conditions, we employ oblique $\mathrm{N}$-curve plot to identify the temporal traffic patterns. $\mathrm{N}$-curves display cumulative vehicle count versus time at different locations (Moskowitz, 1954). For network analysis with multiple origins and destinations, they can represent the cumulative counts of different states. Oblique plots have been extensively utilized in traffic engineering analysis and firstly introduced by Cassidy and Windover (1995). Instead of plotting the cumulative $N$-curve across time a constant background is subtracted at every time period, $q_{0}$. Thus, the plot shows the cumulative flow minus $q_{0} \cdot t$. This simple transformation is for illustration purposes to highlight differences between curves in the same figure, as otherwise differences are not visible due to high values of cumulative variables over time. Note that the accumulation of a state variable can still be measured by the vertical distance between two curves at a specific time instant, but the travel time should now be measured along one of the overlaid oblique lines.

Fig. 8 displays the graphs for the three pricing strategies under discussion: (a) Constant pricing, (b) P1-A and (c) P2. Each graph contains three oblique cumulative $\mathrm{N}$-curve time series for (a) demand for on-street parking (when they enter the network), (b) the transfer flow from running state to cruising state (when cruising starts) and (c) the transfer flow from cruising state to onstreet parking (when they find on-street parking). An oblique distance between the solid black line on the top and the dashed 

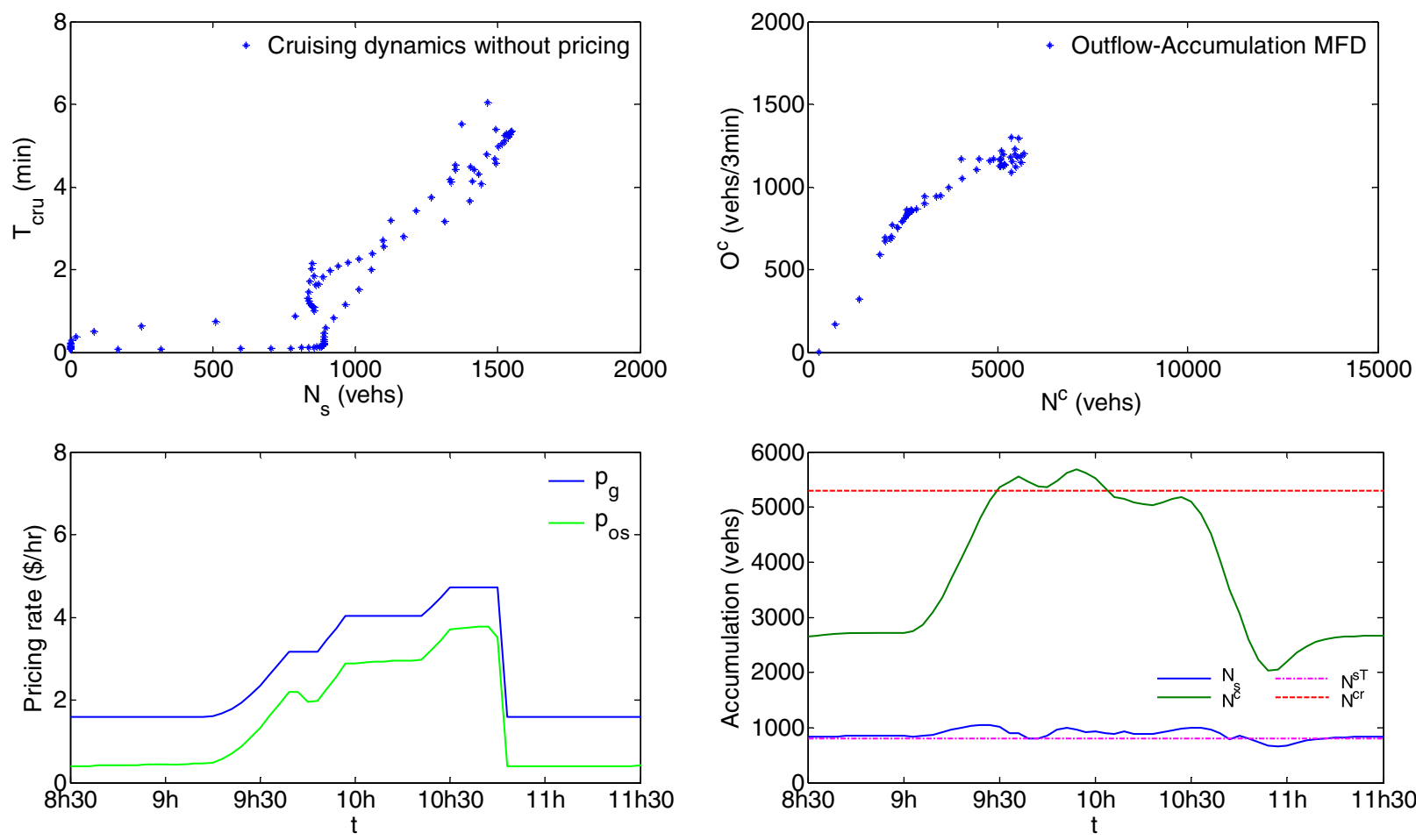

Fig. 7. (a, top left) relationship between cruising delay $T_{\text {cru }}$ and cruising vehicles $N_{s}$ in a non-pricing scenario which is utilized to identify the critical cruising accumulation $N^{s T}$; System performance by strategy P2: (b, top right) the MFD of the center region car network, (c, down left) the prices of $p_{o s}$ and $p_{g}$, and (d, down right) the evolution of accumulations with the desired states in dotted lines: upper red for critical accumulation $N^{c r}$ and magenta for the cruising threshold $N^{N^{T}}$.

Table 2

Comparison of the performances of different pricing schemes.

\begin{tabular}{lllcl}
\hline & Saving in PHT $(\mathrm{h})$ & Total toll $(\mathrm{h})$ & Toll efficiency (ratio: sPHT/TTP) & Ave. cruising delay (min) \\
\hline Base scenario & PHT $=45,337$ & 4660 & 0 & 3.7 \\
Constant pricing & $1211(2.7 \%)$ & 2641 & $45.8 \%$ & 1.9 \\
Strategy P1-A & $6990(15.4 \%)$ & 5857 & $119.5 \%$ & 1.2 \\
Strategy P1-B & $7092(15.6 \%)$ & 9800 & $72.4 \%$ & 1.1 \\
Strategy P2 & $5944(13.0 \%)$ & 7207 & $82.5 \%$ & 1.4 \\
\hline
\end{tabular}

red line in the middle represents the travel time before cruising starts, while the distance between the dashed red line and the solid blue line at the bottom, represents the duration of cruising. Comparing the result under P1-A (Fig. 8(b)) with the one under constant pricing Fig. 8(a), it is clear that both the total delay and the delay caused by cruising are better controlled when P1-A is applied. The reduction in delay is especially high during peak-hour (when travel time before cruising gets larger). The performance under strategy P2 shows similar effectiveness in congestion and cruising control as P1-A. Note that the curves of strategy P2 are not as smooth as the ones of P1-A. This is because traffic states fluctuate during peak-hour due to congestion, while P2 is state-responsive. Nevertheless these results indicate that P2 can maintain an equally efficient mobility level without demand or mode choice information.

\subsection{Efficiency evaluation of the pricing schemes}

In this subsection, the efficiency of the pricing strategies is evaluated. We compare the system costs among the five pricing strategies mentioned above. The following performance indicators are measured: the savings in total person hours travelled (sPHT) compared to the base scenario, the total toll paid (TTP, converted to time unit with a mean value-of-time of $16 \$ / \mathrm{h}$ ), the toll efficiency which is the ratio between sPHT and the corresponding TTP, and the average cruising delay which is the total delay time divided by total amount of on-street parkers. The statistics are summarized in Table 2.

The base scenario applies a flat rate to garage parking only, while on-street is free. On-street parking is highly desired at first, as parking on-street experiences less cost until the cruising time cost increases to garage pricing rate. However due to the large demand for on-street parking, it becomes full and results on average a 3.7 min cruising delay for the later travelers. Users then have to choose garage parking and pay for the fixed high toll. For the studied network, the average travel time per 

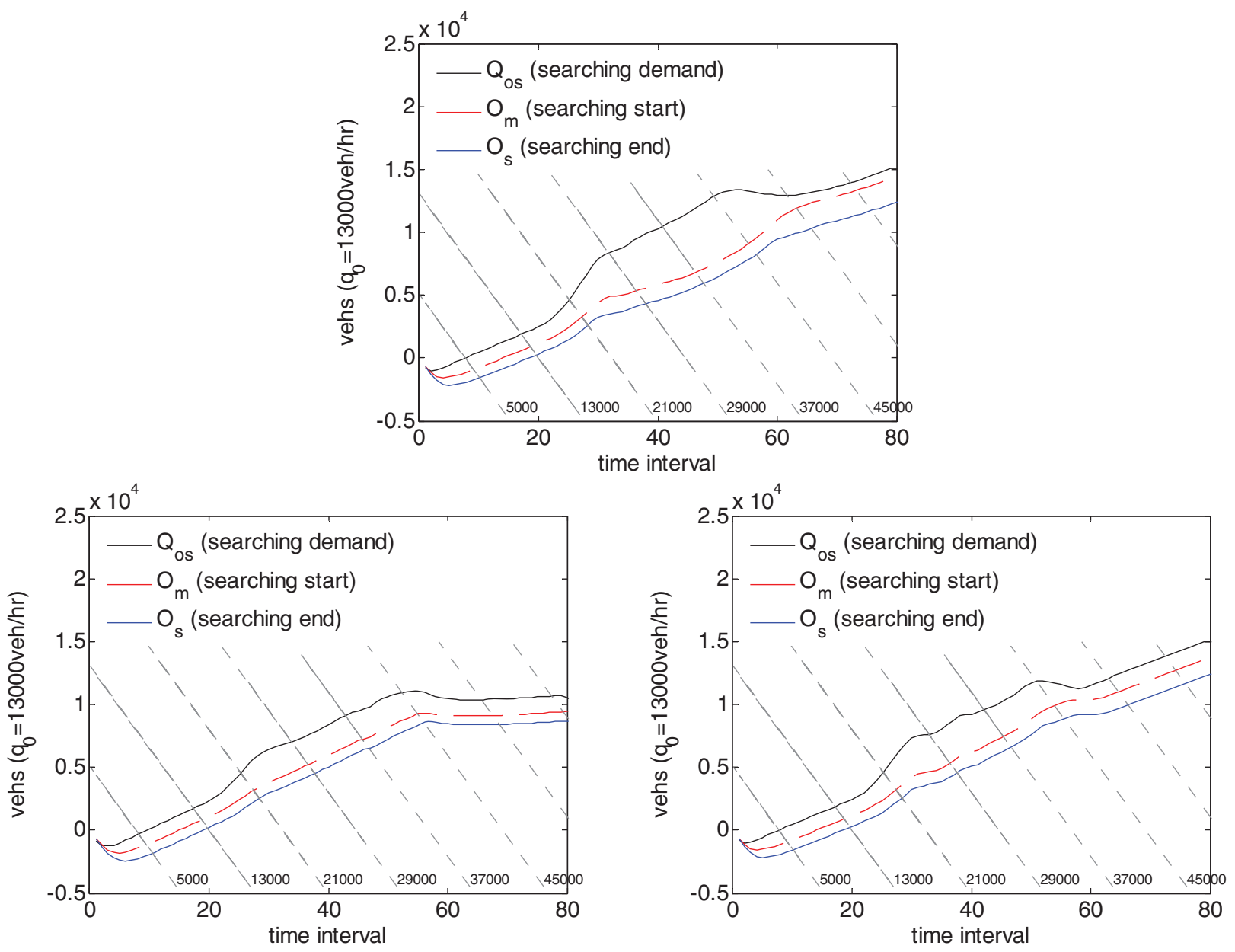

Fig. 8. Oblique plot of the dynamics of the traffic going for on-street parking under the three pricing strategies: (a) Constant pricing, (b, bottom left) Strategy P1-A, and (c, bottom right) strategy P2. Following the oblique dotted gray lines, travel time and cruising time can be estimated.

trip is 6 mins under free-flow condition and around 15 mins when the congestion starts to grow. A 3.7 min cruising time is a relatively considerable amount (more than $50 \%$ of the free flow travel time). Limited improvement in PHT can be found when flat-rate tolls are implemented and optimized for both garage and on-street parking. From an efficiency point of view, however, the constant-pricing strategy makes users pay about two times more than their gain in travel time, reflected by the efficiency rate of $45.8 \%$.

Applying the feedback Strategy P2, it is observed that the PHT significantly decreases by 13\%. The resultant toll efficiency is around $80 \%$ albeit a $120 \%$ can be obtained by utilizing the strategy P1-A. Recall that P1-A is obtained from full optimization by minimizing both the total travel time and the parking toll cost, while P1-B produces optimal prices minimizing only the total travel time. Comparing P1-A and P1-B, while the sPHT is nearly identical, the efficiencies of the two strategies are completely different. This result highlights the importance of considering the total cost for travelers when optimizing pricing schemes. Regarding the difference between P1 and P2, the cruising delays under both P1-type strategies slightly outweigh strategy P2. Regarding the efficiency ratio, the difference can be explained as follows. Since strategy P2 is feedback responsive, controllers (9) and (10) do not exclusively consider congestion evolution. On the other side, strategy P1 is able to predict and adapt traffic conditions and set optimal tolls to trigger an early mode shift as the whole optimization horizon is considered. As mentionedabove, a slight mode shift during the on-set of peak-hour could lead to substantial improvement. While strategy P1 is difficult to implement in reality as it requires future predictions, strategy P2 is a promising pricing scheme for field operation which also leads to an improved system performance, with respect to cruising delay and toll efficiency. Under P2, the prices are determined based on real-time monitoring and the required data are easily observable with the existing technologies (e.g. loop detectors, GPS, Bluetooth etc.). A careful analysis of historical data can possibly make this feasible pricing strategy more efficient, through a model predictive control approach where uncertainty of congestion evolution will be treated in the control horizon. This is a challenging but interesting future direction.

Time-dependent parking-rate represents the current mainstream parking price management in urban areas, though the rates may be determined based on different criteria. As aforementioned, the public-owned parking facilities in the city of San Francisco 

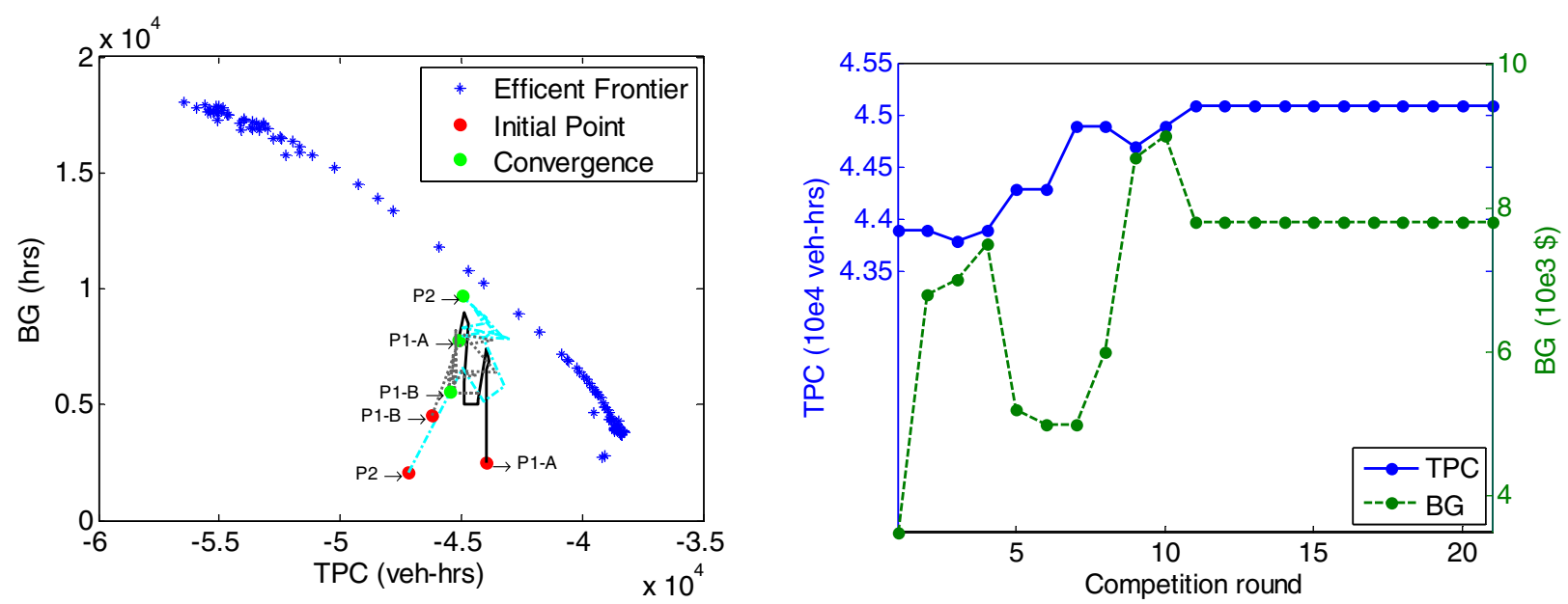

Fig. 9. (a) The efficient frontier between maximizing the system performance (minimizing total cost TPC) for the on-street parking operator and maximizing BG the revenue for garage parking operator; (b) The evolution of the costs of TPC and BG over 20 rounds of leader-follower pricing competition starting from the optimal condition under strategy P1-A (the evolution of P1-A, P1-B and P2 are illustrated in (a), where the red dots mark the initial pairs of the competitions while the green dots mark the equilibria). (For interpretation of the references to color in this figure legend, the reader is referred to the web version of this article).

operate demand-responsive parking pricing which updates the prices at a monthly or shorter basis to reduce cruising time. Strategies P2 attempts to employ such pricing mechanism with a second objective to reduce general congestion. The result shows that the strategy indeed effectively reduces the total PHT and average cruising time. Strategy P1-A demonstrates higher efficiency thanks to the opportunities of adapting the changing conditions of both the users and the parking prices.

We also investigate the performance of P2 strategy if users are receiving imperfect information during their mode choices. Thanks to the current development on information technology, travelers may obtain real-time traffic information via smartphone applications, advanced traveler's systems, radios, etc. Provided the most updated traffic information, smart mode choices (including parking facility choice) can be made to avoid extra travel cost. In practice, however, users may not be able to make choices rationally based on the most recent travel cost. A certain time lag exists between congestion and information propagation and the reaction from the users who enter the network. To this end, we examine if our pricing schemes can adapt to this time lag. We create scenarios where travelers make mode choices based on instantaneous travel and cruising times estimated in the beginning of their trip versus the actual travel time experienced. We obtain a constant pricing through optimization, and a P2 pricing. Comparing to the scenarios where users determine mode choices based only on the current condition (as shown in Table 2), larger amounts of toll are required to control the level of congestion. The mode share of buses falls down about $5 \%$ during the on-set of congestion while rises about 10\% during the off-set of congestion, as users realize the change of travel condition much later. As a result, the maximum accumulations are higher and congestion preserves longer. The efficiency ratios for constant pricing and P2 are 40\% and 75\%, respectively. Evidently, the reduction in efficiency ratio is small. These results imply that though users may not make smart choices in reality due to the lack of information on congestion, feedback pricing indeed can serve as a strong management measure to minimize extra congestion.

\subsection{Parking pricing competition}

Now let us discuss the system performance in a competitive environment where the two parking operators are noncooperative. Firstly, we apply a standard bi-objective optimization procedure to obtain the efficient frontier satisfying both objective functions (11a) and (11b). Two scale parameters valued $(0,1)$ are given to the two objective functions respectively, where the sum of the two parameters equals to 1 . A single-objective optimization is then formulated and different Pareto optimal solutions are produced.

The blue scatter curve in Fig. 9(a) below displays the efficient frontier of this problem. Given this efficient frontier the potential combination of management pricing policies can be readily estimated. Secondly, we illustrate the evolution of competition as described in Fig. 9(b) starting from the three strategies P1-A, P1-B and P2, as initial points, which are below the efficient frontier in Fig. 9(a). The complex dynamics of the developed problem does not allow for analytical derivations of Stackelberg equilibrium. Nevertheless, it is expected that a solution close to the equilibrium is obtained with small error (even if existence or uniqueness cannot be proved). The red dots mark the solutions obtained in the previous subsection while the green dots indicate the point of convergence. It is interesting to observe that competition can help both operators achieve larger benefit if the competition originates from P1-B and P2. If the competition starts from P1-A, the garage operator would end up benefiting from the competition. Meanwhile, the city operator would get slightly worseoff. Detailed evolution of the competition under P1-A is displayed in Fig. 9(b), which shows that a long-term convergence (agreement) can be reached for the two operators after approximately 

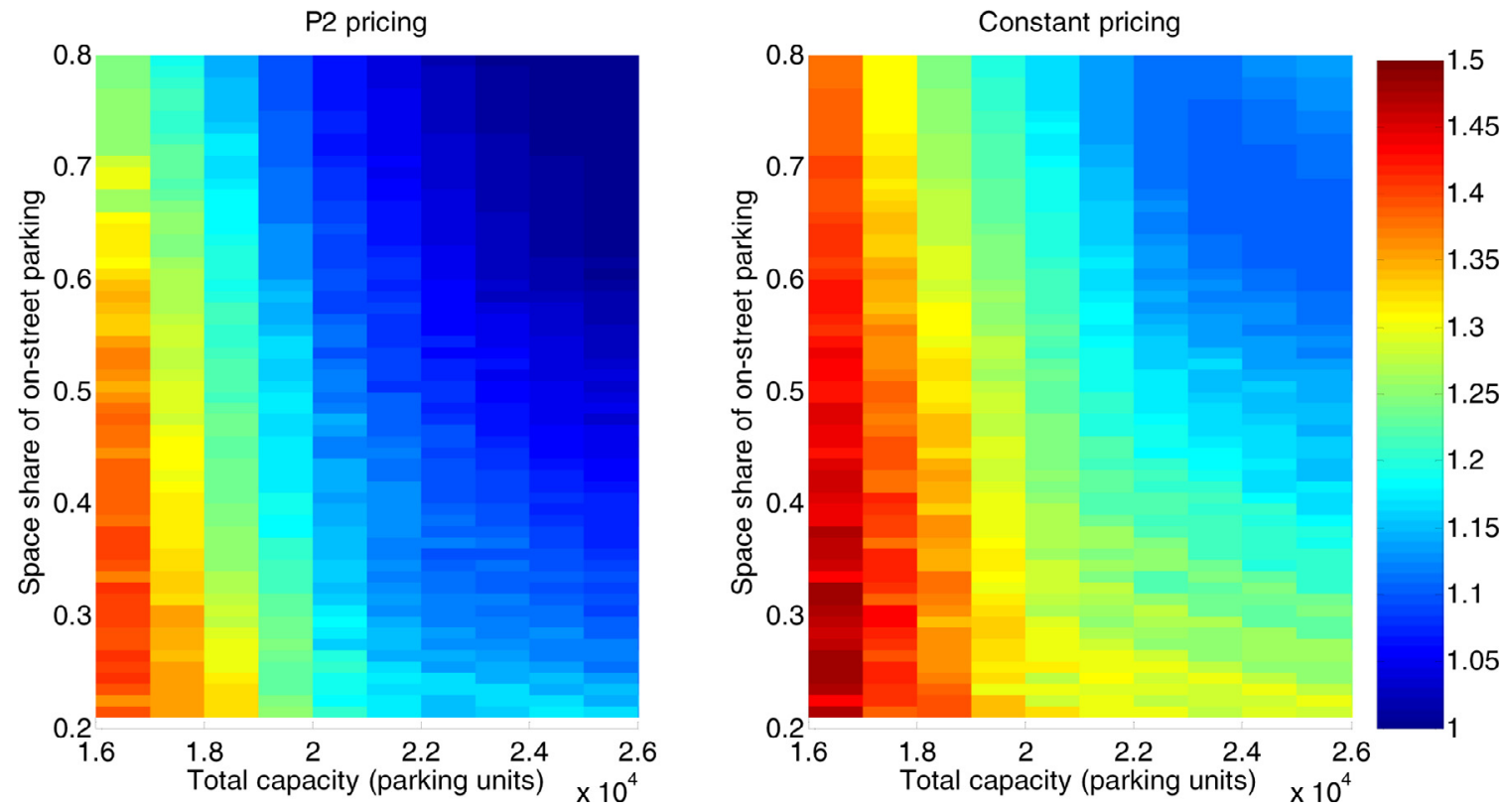

Fig. 10. The contour plots of the normalized PHT values for (a, left) P2 strategy and (b, right) constant pricing under different parking capacity splits between on-street and garage, and different total parking capacities.

10-15 iterations. While the competition converges in solutions close to the efficient frontier, governmental regulations or garage subsidization could help the system to operate at a lower TPC value.

\section{Policy discussion on storage capacity and parking duration}

In the final part of the paper, we provide a discussion on how storage capacity of parking and limit of parking duration influence the dynamics of a multimodal system and how pricing can serve as a supplementary policy to parking space management. Recent research toward this direction can be found in Arnott et al. (2015) who evaluate the effect of on-street parking capacity, duration and pricing on the cruising behavior in congested city centers. In our analysis, we show that urban networks can be operated by proper pricing strategies. In particular, our discussion demonstrates that pricing functions powerfully even when parking storage capacity decreases and cruising delay increases. We will focus on the performance of the P2 strategy which employs the feedback congestion-responsive controller for determining a timely-dynamic pricing. While P1-A functions superiorly under fixed demand scenario, it should be highlighted that the P2 strategy deals with congestion more effectively when demand is uncertain and fluctuates, which implies a substantial potential for practical applications. Our numerical tests confirm this statement. The discussion of this section will provide policy indications on urban parking management through pricing and space allocation.

\subsection{Capacity share between on-street and garage parking}

Up to now, we assume unlimited storage capacity for garages while fixed capacity for on-street parking. In this subsection, we attempt to extend our analysis on paring capacity, and to investigate the impact of different capacity share between the two parking facilities on traffic performance and pricing.

To this end, we set up a base scenario where the total parking space capacity accommodates 24,000 vehicle units, including 19,000 units for on-street parking and 5000 units for garage. We examine system performances of different cases in two dimensions, where we vary: the unit split of the two parking facilities (percentage of the units), and the total space units (total capacity). Please note that when the parking units of on-street decreases, we consider a fixed percentage of these spaces becomes available for road space usage, e.g. more lanes or wider lanes. In another word, the total space of road and on-street parking is assumed to be constant.

We apply P2 strategy and constant pricing to each of the cases, and estimate the total travel time (PHT). The results are illustrated by contour plots and displayed in Fig. 10, where the PHTs are projected over the (total capacity, capacity split) plane. Recall that the $(24,000,80 \%)$ is the base case. We assign value 1 to the PHT of the base case, then normalize the PHTs of all cases by dividing the PHT value to the one of the base case.

The following observations should be highlighted from Fig. 10: (i) When on-street parking capacity is limited, P2 strategy is much more effective than the constant pricing. Note that a case with $(20,000,60 \%)$ decreases the number of on-street spots by $37.5 \%$ (from 19,200 to 12,000 spots) and the total spots by $20 \%$, but with the efficient P2 pricing, network delays increase by 

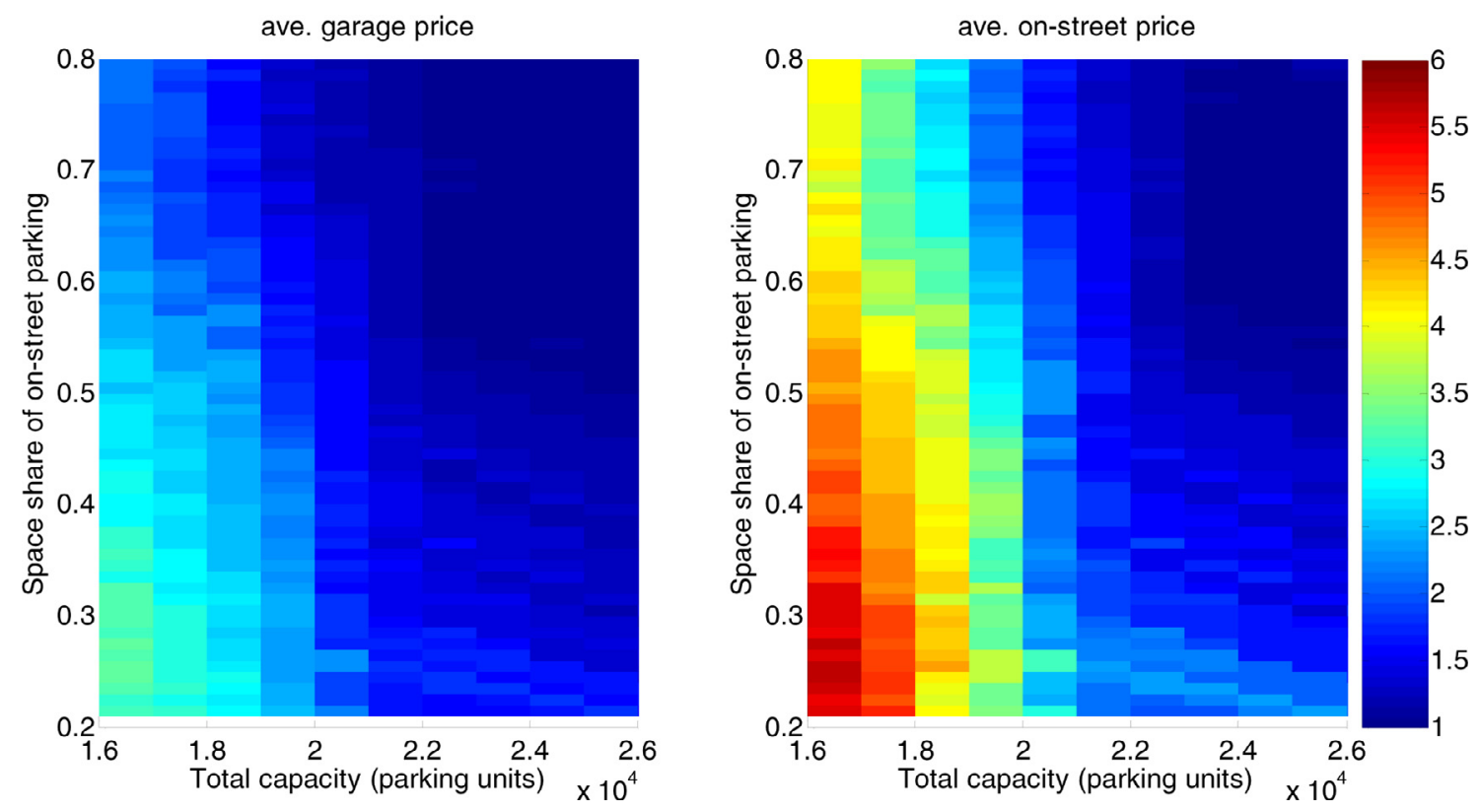

Fig. 11. The contour plots of the normalized average prices during peak-hour of P2 strategy, (a, left) garage parking and (b, right) on-street parking.

only 5\%. For the constant pricing case, delays increase by 30\%; (ii) Applying P2 strategy, there exists an rigid lower bound for the parking units of on-street (the boundary line between the light and dark blue), beyond which the total PHT does not change over the total parking capacities; (iii) Beyond the frontier, increasing the storage capacity does not improve traffic conditions. This holds even when higher prices are enforced; and (iv) PHT increases significantly when the total capacity decreases below a certain level, especially when the share of on-street parking units is low. Furthermore, the resultant mode shift indicates that travelers prefer to switch from on-street parking to garage parking, rather than from cars to buses. For example, when capacity share of on-street drops from $80 \%$ to $20 \%$, only $10 \%$ of travelers change modes to buses while nearly $60 \%$ of the car users switch to garage parking.

In Fig. 11, the contour plots of the average prices during peak-hour are displayed, when P2 strategy is applied. It can be seen that, the prices for both facilities become high when (i) the total capacity decreases, which is logical as finding parking is more and more time-consuming and costly; and (ii) when the share of on-street parking decreases, in particular the on-street parking price increases significantly, which is also reasonable in order to keep the PHTs as low as possible. Note that in situations of limited parking, on-street parking might be more expensive than garage parking. We will show in more details how traffic dynamics and system performances are influenced under such high prices, in a later subsection.

A few policy indications can be interpreted from these results. Firstly, smart parking management should combine capacity planning with pricing, thus saving an important fraction of the limited urban space serving the transportation system. Many cities attempt to build garages in the urban centers, aiming at attracting parking demand and reducing cruising and congestion. Our results indicate that this is not always the proper decision. It is important for cities to identify a possible optimal frontier, and then adjust the share of the capacity split based on their goal of mobility level. Secondly, the improvement in the service of the alternative modes is necessary for the success of parking management. For example if the total collected toll can be utilized to enhance the performance of the buses, pricing would trigger even higher mode shift and achieve greater efficiency in reducing general delay as well as cruising delay. Recall that, we do not allocate the on-street parking capacity fully to road spaces, which means that those spaces can be possibly utilized for infrastructure of pedestrians and cyclists (that can further reduce congestion).

As shown in the previous subsection, the magnitude of storage capacity significantly influences the prices of on-street parking. The dynamic pricing strategy P2 is able to respond to capacity limitations and demand fluctuations, in order to help maintain certain mobility levels (e.g. not exceeding a certain total PHT). Dynamic schemes have already been implemented in cities, such as in the downtown of San Francisco. Shoup (2006) argued that many American cities underprice on-street parking, where the rationale is to use obligatory cruising to rule out user's preference on on-street parking. On-street parking is priced cheap on purpose, attracting large amount of demand. Eventually all on-street parking will be full or highly occupied such that there will be no incentive for people to cruise but to park in garage. Such policy aims at maintaining a high utilization rate of parking space (e.g. as long as there is an empty space, it will be filled immediately), and redistributing users over different modes through a day-to-day type of learning. However, cruising delay cannot be eliminated or significantly decreased under such pricing scheme. Therefore, underpricing may not be as efficient as strategies P1 and P2. This is because underpricing on-street parking brings longer cruising, and enforces the users pay higher parking prices as they need to go to garage. On the other hand, overpricing 

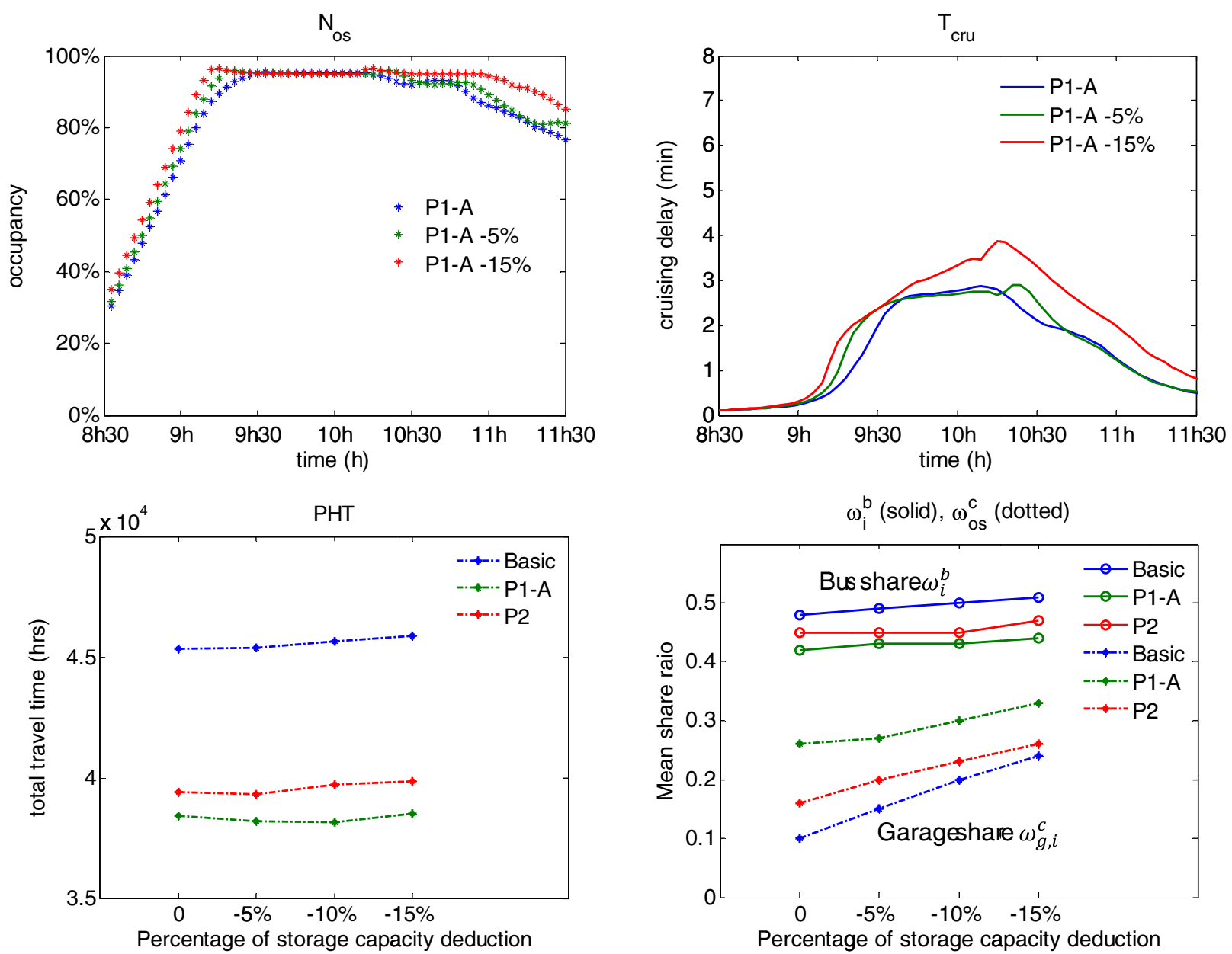

Fig. 12. Evolution of (a, top left) on-street parking occupancy over time, and (b, top right) cruising delay over time, under different on-street parking capacities; Comparison of (c, down left) total cost TPC and total travel time PHT over different on-street parking storage capacities, and (d, down right) average mode share of bus, by solid lines, and average share of garage parking over different on-street parking storage capacities, under three pricing scenarios: Basic, P1-A and P2.

(e.g. by P1-B) is indeed effective in congestion reduction (as via mode shift). However it cancels out the saving from travel time and results a higher total user cost.

Some policy makers might advocate allocating more space to on-street parking in order to mitigate delay caused by cruising. However this may not be an optimal strategy. From parking operational point of view, we have confirmed this point in the previous section. From a global city planning point of view, as urban road space is limited in the city center, allocating more space for parking means for example demanding space from the dedicated bus lanes. Thus, space-allocation and -reallocation should take into account the space competition among different modes that may share the total road space, and quantitative evaluations shall be carried out, e.g. through an optimization process proposed in Guler and Cassidy (2012) for link level or in Zheng and Geroliminis (2013) for network level. Space re-allocation may be expensive to implement or even an infeasible solution. Furthermore, adapting space allocation to demand fluctuation is difficult and unsustainable. Pricing such as the ones designed in this paper therefore can serve as a supplementary strategy under capacity limitation, by triggering mode choice shift including parking facility choices.

\subsection{Storage capacity of on-street parking}

To this end, we investigate in more details how parking capacity influences traffic dynamics, mode share, and pricing. In particular, we focus on on-street parking. We utilize the base scenario where the total parking capacity and split are $(24,000$, $80 \%$ ), and obtain the system performances under another four scenarios: no change (denoted as $0 \%$ ), $5 \%, 10 \%$ and $15 \%$ deduction of on-street parking space. The dynamics under pricing strategy P1-A are simulated and results of three scenarios are displayed in Figs. 12(a) and 12(b). P1-A is re-optimized for each of the scenarios. It can be seen that the maximum occupancy in all scenarios goes up to $90 \%$. By comparing the slopes of the three curves between occupancy values of $30 \%$ and $90 \%$, we observe that storage capacity influences how fast the maximum occupancy rate is reached. The more the capacity is provided, the less drastic the 

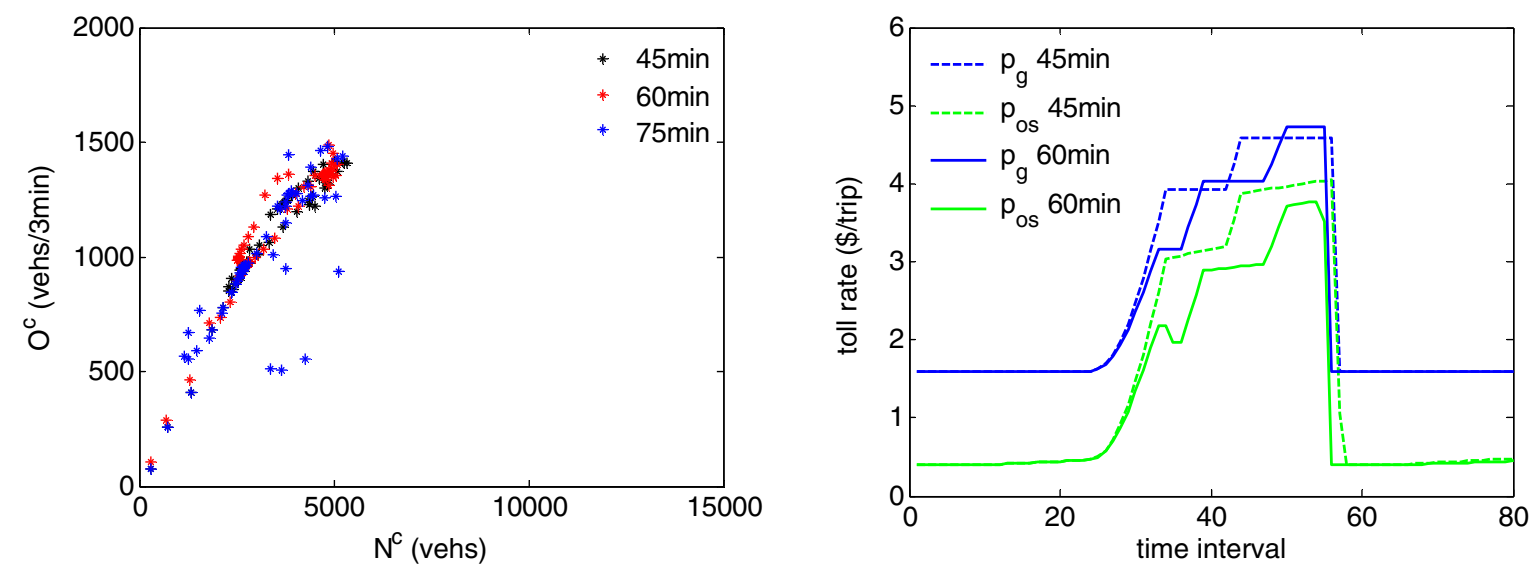

Fig. 13. The MFDs of different parking duration limits (a, left) under the dynamic strategy P2, and (b, right) comparison of the pricing rates of P2 between cases of parking duration $45 \mathrm{~min}$ and $60 \mathrm{~min}$.

occupancy increases. If we compare the reductions of occupancy rate after time $11 \mathrm{~h}$ for the three scenarios, we can find that the larger the capacity, the faster the system recovers from the overcrowding of on-street parking during the off-set of the peak hour. Fig. 12(b) compares cruising delay under the three scenarios. As the capacity decreases, car-users experience higher cruising delays for longer duration, but not too longer. Low on-street availability causes longer cruising distance. Travel speed decreases, which is a result of the accumulation of cruising vehicles. Consequently, larger cruising delay would be experienced. Though the change of occupancy follows a similar pattern, it is shown in the figure that the average cruising delay for case "P1 -15\%" is about 0.5 min larger than that of "P1" and "P1 -5\%". Furthermore, the cruising-for-parking phenomenon lasts longer when capacity decreases, which correspond to what we observed in Fig. 12(a).

Then we estimate total travel time PHT, the average mode share of bus and the average share of garage parking for the four scenarios mentioned above. We compare these performance indicators under three pricing strategies: Basic pricing (recall this is a constant pricing without optimization), P1-A pricing, and P2 pricing. The results are illustrated in Fig. 12(c). It can be observed that PHT vary within $4 \%$ as storage capacity gradually decreases, while the total cost TPC follows the same pattern (not shown). This is promising result as it indicates that (I) proper pricing can maintain equivalent of level system performance even when storage capacity is reduced by certain percentage, and (II) the real-time pricing strategy P2 shows similar efficiency to strategy P1-A. If this space would be allocated to improve public transport services, further improvements and higher public transport mode shares could be observed. What is interesting about argument (I) is that one may expect PHT significantly increase when capacity decreases and Fig. 12(b) actually confirms that the cruising time becomes larger. This indeed would be the case, if users had no other mode choices but car with on-street parking. As proper pricing redistributes effectively the demand for car usage and on-street parking usage overall system performance is not influenced. Fig. 12(c) compares the average mode share of bus $\left(\omega_{i}^{b}\right)$ and the average garage share $\left(\omega_{g, i}^{c}\right)$. While both shares exhibit a tendency of increase, garage attracts more trips than public transport. This could be influenced if the decrease for on-street parking would be better utilized for public transport. As garage parking requires no cruising, saving in PHT can be expected. This saving would compensate the increase in cruising delay for users still going for on-street parking, thus explains the slight variation observed in Fig. 12(c).

\subsection{Allow a shorter or longer parking duration?}

The amount of available parking spaces depends not only on the physical storage capacity of the parking facilities, but also on parking duration which regulates the amount of time a user can occupy a parking space. With users reaching their duration limit and re-departing, available parking spaces are generated dynamically. A shorter duration enforces a higher departure rate from parking spaces, thus leading to more accessible parking conditions. However on the other hand, such conditions can attract higher amount of car users that result higher cruising flow and congestion. A relevant policy question should be answered then is how parking duration limit affects the flow dynamics.

To this end, we modify the parking duration limits for on-street parking by $\pm 25 \%$ (45 min and 75 min), and we estimate the P2 prices for these two cases. The resultant MFDs are displayed in Fig. 13(a), together with the MFD of the 60 min-case (Fig. 7(b)). Overall, the MFDs show that congestion can be sufficiently controlled (accumulations are below the critical value), under the different parking duration restrictions. The total PHTs for the three cases are which are nearly identical, within $0.5 \%$ difference. To achieve this, higher pricing rates are applied in the cases of $45 \mathrm{~min}$ and $75 \mathrm{~min}$, comparing to the base case of $60 \mathrm{~min}$. Under short-duration allowance, users tend to prefer on-street parking because parking spaces are more frequently available. Higher pricing rates are required in order to force mode shift to avoid overcrowding of cruising and congestion. The time series of the pricing rates displayed in Fig. 13(b) verify this statement. While when long-duration is allowed, higher pricing is obtained as well. For the sake of visibility, we do not plot the pricing time series of the 75 min case, which are similar to the case of 45 min. 
For both cases, higher mode share of bus $\omega_{i}^{b}$ are also found (47\%, 45\% and 50\% for $45 \mathrm{~min}, 60 \mathrm{~min}$, and 75 min respectively), which is due to the augment of travel cost from pricing and cruising time.

Note that some scatters can be observed below the MFD of $75 \mathrm{~min}$. It is caused by the extremely low on-street parking availability, which limits the trip completion rate of the whole region.

Note also that we focus on the scenarios of fixed parking duration (similar consideration can be found e.g. in Arnott et al. (2015) in our study. Integrating stochasticity in our model, i.e. assuming parking duration follows a certain distribution, is more challenging as the first in-first out rule is violated. It however would complicate the system dynamics and exceed the scope of this study. We aim to investigate this subject more carefully in future work.

\section{Conclusion}

In this study, we proposed a macroscopic approach for modeling multi-modal traffic system with parking limitation and cruising-for-parking flow. Parking limitation was integrated in the developed multi-modal system model, where vehicles need to cruise for parking before reaching their destination. The time of cruising was estimated by assuming the probability of finding a parking space follows a geometric distribution and depends on the dynamic parking availability. The effect of cruising on the global performance, e.g. the average speed, was also captured, by the MFD dynamics. A case study was carried out in a two-region bi-modal network. Two parking choices were considered: (i) limited on-street parking requiring cruising, and (ii) unlimited garage parking with higher parking fee but no cruising cost. We then utilized the system model to test two networklevel parking pricing strategies. Strategy P1 was obtained through optimization of the total travel cost (PHT + parking fee, P1-A) or total travel time (PHT only, P1-B). Under this strategy, the prices were determined with long-term impact taking into account and therefore the pricing scheme is highly efficient. Strategy P2 adapted a feedback-type controller for determining the parking price, which was congestion- and parking availability-dependent. This pricing strategy helped maintain traffic performance at desired (controlled) levels. Furthermore, it was promising that this strategy, which can be implemented in real-time, was closely efficient as Strategy P1. Following these results, we investigated the competition behavior and system performance, when the authorities of on-street and garage parking belong to different operators who manage the prices with different objectives. Results of cooperative-competition via a bi-objective optimization and a selfish-competition via a bi-level optimization were presented. It was found that competition could not improve the benefit of neither of the operators, compared to cooperative strategies, such as P1 or P2. Finally, we discussed the impact of the on-street parking storage capacity on system dynamics. We showed even if the capacity decreases for 30\%, pricing strategy such as P2 can keep PHT at similar level. We also found that system performance could be well maintained under capacity reduction, when the proposed pricing strategies were applied and triggered mode shift from car to bus, and from on-street parking to garage. Pricing can support smart space allocation through a comprehensive optimization procedure, where the effect of multi-mode traffic is considered.

On-going work extends the investigation on the efficiency of parking management under pricing competition. Not all aspects of modeling have been validated until now due to lack of detailed data, as for example the parking model where empirical study will be carried out for model verification and parameter calibration. Empirical studies should be also planned on the identification of the start of cruising, utilizing data such as GPS records. Furthermore, the current treatment of cruising is dimensionless, meaning that the cruising cost is estimated without identifying the detailed routes of cruising. Such consideration aims at the average cost at system level and ignores the cruising possibilities at disaggregated level. Nevertheless, large spatial heterogeneity is not expected given the spatial correlations in the distribution of congestion and well-defined bi-modal MFD can be developed for more complex city structures. A multi-region analysis with a consideration of multiple parking facilities with limited availability should be a research priority. One additional complexity might be that multiple regions with parking limitations would require the addition of a location choice model, with additional cost components (accessibility, distance from the destination, etc.).

\section{Acknowledgment}

This research was partially supported by ERC Starting Grant "METAFERW: Modeling and controlling traffic congestion and propagation in large-scale urban multimodal networks". The authors would like to thank the anonymous reviewers for their constructive comments.

\section{References}

Aboudolas, K., Geroliminis, N., 2013. Perimeter and boundary flow control in multi-reservoir heterogeneous networks. Transportation Research Part B 55, 265281.

Anderson, S., de Palma, A., 2004. The economics of pricing parking. Journal of Urban Economics 55, 1-20. Anderson, S., de Palma, A., 2007. Parking in the city. Regional Sciences 86 (4), 621-632.

Arnott, R., 2006. Spatial competition between parking garages and downtown parking policy. Transport Policy 13 (6), 458-469.

Arnott, R., Inci, E., 2010. The stability of downtown parking and traffic congestion. Journal of Urban Economics 68 (3), $260-276$.

Arnott, R., Inci, E., Rowse, J., 2015. Downtown curbside parking capacity. Journal of Urban Economics 86, 83-97.

Arnott, R., Rowse, J., 1999. Modeling parking. Journal of Urban Economics 45, 97-124.

Arnott, R., Rowse, J., 2009. Downtown parking in auto city. Regional Science and Urban Economics 39 (1), 1-14.

Bagchi, A., 1984. Stackelberg differential games in economic models. Springer Verlag 1984-Business\&Economics, New York.

Ben-Akiva, M., Bierlaire, M., 1999. Discrete choice methods and their applications to short term travel decisions. Handbook of Transportation Science 23, 5-33.

Cassidy, M., Windover, J., 1995. Methodology for assessing dynamics of freeway traffic flow. Transportation Research Record 1484, 73-79. 
Cao, J., Menendez, M., 2015a. Generalized effects of on-street parking maneuvers on the performance of nearby signalized intersections. Transportation Research Record (in press).

Cao, J., Menendez, M., 2015b. A parking-state-based transition matrix of traffic on urban networks. Transportation Research Part B 7, 149-169.

Chiabaut, N., 2015. Evaluation of a multimodal urban arterial: The passenger macroscopic fundamental diagram. Transportation Research Part B doi:10.1016/j. trb.2015.02.005, (in press).

Chiabaut, N., Xie, X., Leclercq, L., 2014. Performance analysis for different designs of a multimodal urban arterial. Transportmetrica B 2 (3), 229-245.

Forsgerau, M., dePalma, A., 2013. The dynamics of urban traffic congestion and the price of parking. Journal of Public Economics 105, $106-115$.

Gallo, M., D’Acierno, L., Montella, B., 2011. A multiplayer model to simulate cruising for parking in urban areas. Transport Policy 18 (5), $735-744$.

Geroliminis, N., Daganzo, C., 2008. Existence of urban-scale macroscopic fundamental diagrams: some experimental findings. Transportation Research Part B 42 (9), 759-770.

Geroliminis, N., 2009. Dynamics of peak hour and effect of parking for congested cities. In: Proc. of the 88th Annual Meeting of Transportation Research Board. Washington D.C., USA.

Geroliminis, N., 2015. Cruising-for-parking in congested cities with an MFD representation. Economics of Transportation 4 (3), $156-165$.

Geroliminis, N., Boyaci, B., 2012. The effect of variability of urban systems characteristics in the network capacity. Transportation Research Part B 46 (10), 16071623.

Geroliminis, N., Levinson, D., 16-18 July, 2009. Cordon pricing consistent with the physics of overcrowding. In: Proc. of the 18th International Symposium on Transportation and Traffic Theory (ISTTT). Hong Kong, pp. 219-240.

Geroliminis, N., Zheng, N., Ampountolas, K., 2014. A three-dimensional fundamental diagram for mixed bi-modal urban networks. Transportation Research Part C 42, 168-181.

Godfrey, J., 1969. The mechanism of a road network. Traffic Engineering and Control 11 (7), 323-327.

Guler, I., Cassidy, M., 2012. Strategies for sharing bottleneck capacity among buses and cars. Transportation Research Part B 46, $1334-1345$.

Guo, H., Gao, Z., 2012. Modelling travel time under the influence of on-street parking. Journal of Transportation Engineering 138 (2), $229-235$.

Haddad, J., Ramezani, M., Geroliminis, N., 2013. Cooperative traffic control of a mixed network with two urban regions and a freeway. Transportation Research Part B 54, 17-36.

Haddad, J., 2015. Robust constrained control of uncertain macroscopic fundamental diagram networks. Transportation Research Part C 59, 323-339.

He, F., Yin, Y., Chen, Z., Zhou, J., 2015. Pricing of parking games with atomic players. Transportation Research Part B 73, 1-12.

Horni, A., Montini, L., Waraich, R., Axhausen, K., 2013. An agent-based cellular automaton cruising-for-parking simulation. Transportation Letters 5 (4), 167-175.

Ji, Y., Geroliminis, N., 2012. On the spatial partitioning of urban transportation networks. Transportation Research Part B 46 (10), $1639-1656$.

Keyvan-Ekbatani, M., Yildirimoglu, M., Geroliminis, N., Papageorgiou, M., 2015. Multiple concentric gating traffic control in large-scale urban networks. IEEE Transactions on Intelligent Transportation Systems 16 (4), 2141-2154.

Kosmatopoulos, E., Papageorgiou, M., 2003. Stability analysis of the freeway ramp metering control strategy ALINEA. In: Proc. of the 11th Mediterranean Conference on Control \& Automation. Rhodes, Greece.

Leclercq, L., Chiabaut, N., Trinquier, B., 2014. Macroscopic fundamental diagrams: a cross-comparison of estimation methods. Transportation Research Part B 62, 1-12.

Leclercq, L., Geroliminis, N., 2013. Estimating MFDs in simple networks with route choice. Transportation Research Part B 57, $468-484$.

Leclercq, L., Parzani, C., Knoop, V., Amourette, J., Hoogendoorn, S., 2015. Macroscopic traffic dynamics with heterogeneous route patterns. Transportation Research Part C 59, 292-307.

Li, Z., Huang, H., Lam, W., Wong, S., 2007. A model for evaluation of transport policies in multimodal networks with road and parking capacity constraints. Journal of Mathematic Model and Algorithm 6, 239-257.

Liu, T., Huang, H., Yang, H., Zhang, X., 2009. Continuum modeling of park-and-ride services in a linear monocentric city with deterministic mode choice. Transportation Research Part B 43, 692-707.

Liu, W., Yang, H., Yin, Y., 2014. Expirable parking reservations for managing morning commute with parking space constraints. Transportation Research Part C 44, $185-201$.

Mahmassani, H., Saberi, M., Zockaie, A., 2013. Urban network gridlock: theory, characteristics, and dynamics. Transportation Research Part C 36, $480-497$.

Martens, K., Benenson, I., Levy, N., 2010. The dilemma of on-street parking policy: exploring cruising for parking using an agent-based model. Geo Journal Library 99, 121-138.

Moskowitz, K., 1954. Waiting for a gap in a traffic stream. Proceedings of the Highway Research Board 33, 385-395.

Nocedal, J., Wright, J., 2006. Numerical Optimization, second ed Springer Series in Operations Research, Springer Verlag.

Ortigosa, J., Menendez, M., Tapia, H., 2014. Study on the number and location of measurement points for an MFD perimeter control scheme: a case study of Zurich. EURO Journal on Transportation and Logistics 3 (3-4), 245-266.

Papageorgiou, M., Kotsialos, A., 2002. Freeway ramp metering: an overview. IEEE Transactions on Intelligent Transportation Systems 3 (4), $228-239$.

Qian, Z., Rajagopal, R., 2014. Optimal dynamic parking pricing for morning commute considering expected cruising time. Transportation Research Part C 48, $468-490$.

Qian, Z., Xiao, F., Zhang, H., 2013. Managing morning commute traffic with parking. Transportation Research Part B 46 (7), 894-916.

Ramezani, M., Haddad, J., Geroliminis, N., 2015. Dynamics of heterogeneity in urban networks: aggregated traffic modeling and hierarchical control. Transportation Research Part B 74, 1-19.

Saeedmanesh, M., Geroliminis, N., 2015. Optimization-based clustering of traffic networks using distinct local components. In: Accepted for the 18th IEEE conference on Intelligent Transportation System (ITSC). Gran Canary, Spain.

Shoup, D., 2006. Cruising for parking. Transport Policy 13, 479-486.

Simoni, M., Pel, A., Waraich, R., Hoogendoorn, S., 2015. Marginal cost congestion pricing based on the network fundamental diagram. Transportation Research Part C 56, 221-238.

Van Ommeren, J., Wentink, D., Rietveld, P., 2012. Empirical evidence on cruising for parking. Transportation Research Part A 46 (1), 123-130.

Verhoef, E., Nijkamp, P., Rietveld, P., 1995. The Economics of regulatory parking policies: the (im) possibilities of parking policies in traffic regulation. Transportation Research Part A 29 (2), 141-156.

Vickrey, W., 1969. Congestion theory and transport investment. American Economic Review 59 (2), 251-260.

Yildirimoglu, M., Geroliminis, N., 2014. Approximating dynamic equilibrium conditions with Macroscopic Fundamental Diagrams. Transportation Research Part B 70, 186-200.

Yildirimoglu, M., Ramezani, M., Geroliminis, N., 2015. Equilibrium analysis and route guidance in large-scale networks with MFD dynamics. Transportation Research Part C 59, 404-420.

Yin, Y., 2002. Multi-objective bi-level optimization for transportation planning and management problems. Journal of Advanced Transportation 36 (1), 93-105 .

Yousif, S., Purnawan, P., 2004. Traffic operations at on-street parking facilities. Proceedings of the Institution of Civil Engineers-Transport 157 (3), $189-194$.

Zheng, N., Waraich, R., Axhausen, K., Geroliminis, N., 2012. A dynamic cordon pricing scheme combining the Macroscopic Fundamental Diagram and an agentbased traffic model. Transportation Research Part A 46 (8), 1291-1303.

Zheng, N., Geroliminis, N., 2013. On the distribution of road space for urban multimodal congested networks. Transportation Research Part B 57, 326-341.

Zhou, X., Mahmassani, H., Zhang, K., 2008. Dynamic micro-assignment modelling approach for integrated multimodal urban corridor management. Transportation Research Part C 16 (2), 167-186. 